\title{
Unification of [FeFe]-hydrogenases into Three Structural and Functional Groups
}

4

5 Saroj Poudel (saroz189@gmail.com) ${ }^{1}$, Monika Tokmina-Lukaszewska

6 (tokminalukas@gmail.com) ${ }^{2}$, Daniel R. Colman (daniel.colman@montana.edu) ${ }^{1}$, Mohammed

7 Refai (refai1982@gmail.com) ${ }^{2}$, Gerrit J. Schut (gerti@uga.edu) ${ }^{3}$, Paul W. King

8 (Paul.King@nrel.gov) ${ }^{4}$, Pin-Ching Maness (PinChing.Maness@nrel.gov) ${ }^{4}$, Michael W.W.

9 Adams (adams@bmb.uga.edu) ${ }^{3}$, John W. Peters (john.peters@ chemistry.montana.edu) ${ }^{2}$, Brian

10 Bothner (bbothner@chemistry.montana.edu) ${ }^{2}$ and Eric S. Boyd (eboyd@ montana.edu) ${ }^{1^{*}}$

11

$12{ }^{1}$ Department of Microbiology and Immunology, Montana State University, Bozeman, MT 59717

13

14

15

16

17

18

19

20

21

22

23

24

25

26

27
Eric S. Boyd ( $\underline{\text { eboyd@montana.edu) }}$

Department of Microbiology \& Immunology

Montana State University

PO Box 173520

Bozeman, MT 59717

Phone: (406) 994-7046

Fax: (406) 994-4926 


\section{ABSTRACT}

Background. [FeFe]-hydrogenases (Hyd) are structurally diverse enzymes that catalyze the reversible oxidation of hydrogen $\left(\mathrm{H}_{2}\right)$. Recent biochemical data demonstrate new functional roles for these enzymes, including those that function in electron bifurcation where an exergonic reaction is coupled with an endergonic reaction to drive the reversible oxidation/production of $\mathrm{H}_{2}$.

Methods. To identify the structural determinants that underpin differences in enzyme functionality, a total of 714 homologous sequences of the catalytic subunit, HydA, were compiled. Bioinformatics approaches informed by biochemical data were then used to characterize differences in inferred quaternary structure, HydA active site protein environment, accessory iron-sulfur clusters in HydA, and regulatory proteins encoded in HydA gene

40 neighborhoods.

Results. HydA homologs were clustered into one of three classification groups, Group 1

42 (G1), Group $2(\mathrm{G} 2)$, and Group 3 (G3). G1 enzymes were predicted to be monomeric while those 43 in G2 and G3 were predicted to be multimeric and include HydB, HydC (G2/G3) and HydD

44 (G3) subunits. Variation in the HydA active site and accessory iron-sulfur clusters did not vary 45 by group type. Group-specific regulatory genes were identified in the gene neighborhoods of 46 both G2 and G3 Hyd. Analyses of purified G2 and G3 enzymes by mass spectrometry strongly 47 suggests that they are post-translationally modified by phosphorylation. presence of both HydB and HydC in Hyd complexes, rather than by variation in HydA. 
General Significance. This classification scheme provides a framework for future

51 biochemical and mutagenesis studies to elucidate the functional role of Hyd enzymes.

53 Abstract word limit: 250. Word count: 249

54

55

Key words: [FeFe]-hydrogenase, hydrogen, electron bifurcation, post-translational modification, regulation, bioinformatics

57

\section{INTRODUCTION}

Hydrogenases catalyze the reversible reduction of protons to hydrogen $\left(\mathrm{H}_{2}\right)$ gas using a variety of electron donors and acceptors. The three primary variants of hydrogenase, termed

$62[\mathrm{NiFe}]-,[\mathrm{FeFe}]-$, and $[\mathrm{Fe}]-$ hydrogenases, are evolutionarily distinct and differ in the composition 63 of their active site metal clusters [1-10]. Of the three variants, [FeFe]-hydrogenases (Hyd) are of

64 substantial interest for biotechnology and bioenergy purposes due to their high rate of proton

65 reduction [11-13]. Hyds are widely distributed in Bacteria and also have been detected in a

66 number of Eukarya but have yet to be identified in Archaea [14-17]. Hyds vary in their cellular

67 localization which corresponds with differing functionalities. For instance, Hyds can be localized

68 in the periplasm or the cytoplasm and can be either membrane-bound or soluble [18-24].

69 Periplasmic Hyds are primarily involved in $\mathrm{H}_{2}$ uptake while membrane-bound or soluble

70 cytoplasmic Hyds are involved in reversible oxidation of $\mathrm{H}_{2}$ [18-22, 24].

71 
At a minimum Hyds comprise a catalytic subunit, HydA, which contains a conserved

$73 \sim 350$ residue $\mathrm{H}$-cluster domain that harbors the ligands for the novel active site iron-sulfur

74 cluster. The active site iron-sulfur cluster consists of a [4Fe-4S] cluster bridged to a $2 \mathrm{Fe}$

75 subcluster via a cysteine thiol $[1,11,17,25]$. In addition to the H-cluster domain, HydA often contains N-terminal (F-cluster) and C-terminal (C-cluster) domains that add flexibility to the

77 enzyme and that modulate electron flow to and/or from the active site H-cluster $[11,14,16,17$,

$7825,26]$. The simplest form of HydA includes those identified in green algae, such as

79 Chlamydomonas reinhardtii, which generally only contains the H-cluster domain [27-32]. More

80 complex forms of HydA are more common and possess several additional iron-sulfur clusters,

81 including those with both the F- and C-clusters $[2,11,33]$. In addition to F- and C-cluster

82 mediated functional flexibility, HydA can exist in multiple isoforms with different quaternary

83 structures, including those that are monomeric and those that are multimeric $[14,16,17,26,34]$.

84 Multiple HydAs of variable quaternary structure can be present in a single genome [22, 35-37]

85 where they form complexes with paralogs of the large and small subunits of NADH

86 dehydrogenase (referred to as HydB and HydC, respectively) [21, 35-39].

87

Recent biochemical studies indicate that several soluble, cytoplasmic and, multimeric

89 Hyds are capable of electron bifurcation, a novel mechanism of energy conservation that

90 involves the simultaneous reduction or oxidation of two electron acceptors or donors in an

91 enzyme complex, whereby a thermodynamically favorable exergonic reaction drives a

92 thermodynamically unfavorable endergonic reaction [21, 35, 37-42]. Soluble cytoplasmic Hyds

93 identified from Acetobacterium woodii [21], Clostridium autoethanogenum [40], Moorella

94 thermoacetica [36] and Thermotoga maritima [35] are all capable of electron bifurcation [38, 
39]. The trimeric Hyd from T. maritima (i.e. HydABC complex) couples the simultaneous oxidation of ferredoxin (Fd) and $\mathrm{NADH}$ to the production of $\mathrm{H}_{2}$ [35]. In contrast, the tetrameric Hyd from A. woodii (i.e. HydABCD complex) couples the oxidation of $\mathrm{H}_{2}$ to the simultaneous reduction of $\mathrm{Fd}$ and $\mathrm{NAD}^{+}$[21]. Interestingly, a trimeric Hyd from the acetogen $M$. thermoacetica functions in vivo to either oxidize or produce $\mathrm{H}_{2}$, contingent on cultivation conditions, by coupling $\mathrm{Fd}$ and $\mathrm{NAD}^{+} / \mathrm{NADH}$ reduction/oxidation [43]. Autotrophically-grown M. thermoacetica cells oxidize $\mathrm{H}_{2}$ by simultaneously reducing both $\mathrm{Fd}$ and $\mathrm{NAD}^{+}$while glucosegrown cells used the same enzyme complex to reduce protons to produce $\mathrm{H}_{2}$ by coupling with the oxidation of Fd and NADH. Conversely, the Hyd from C. pasteurianum (cytoplasmic and soluble) has been shown to not bifurcate electrons, which is consistent with its lack of a full length HydB $[2,11,36]$. Likewise, the 'dimeric' Hyd from Desulfovibrio desulfuricans, which has a truncated HydB that functions in part to target the enzyme to the periplasm, is unlikely to bifurcate $[23,44]$. example, A. woodii [21], Ruminococcus albus [37], and T. maritima [35], among others [14, 16]. The presence of multiple copies of HydA including those that bifurcate and those that do not suggests a need to regulate one or more of the enzymes in order to maximize energy conservation. Indeed, a recent study identified several genes that encode for proteins putatively involved in sensing $\mathrm{H}_{2}$ and in the post-translational modification (PTM) in the gene neighborhood of seven different HydAs (including both monomeric and trimeric proteins) [37].

One of those proteins, HydS, was proposed as a $\mathrm{H}_{2}$ sensor based on the presence of a Per-ARNTSim (PAS) domain, which are widely used to promote protein-protein interactions or signal 
transduction [37, 45]. Moreover, proteins hypothesized to be involved in signal transduction (e.g. serine-threonine kinases/phosphatases and histidine kinases) have been identified in the flanking gene environment of hydA in C. thermocellum and T. maritima [37]. Whether these enzymes are involved in PTM of Hyd proteins and the extent to which they are distributed in the gene neighborhoods of hydA among other microorganisms is not yet known.

In order to develop a robust classification scheme for use in predicting bifurcating and non-bifurcating HydA, we compiled all the available HydAs in completed genomes. Our goal was to build upon and at the same time reconcile past classification schemes $[14,16]$. A comprehensive characterization of the proteins encoded in the neighboring genomic regions of hydA was also conducted using bioinformatics approaches. This information was then used to develop classifications of Hyd that unify patterns in enzyme diversity at the level of primary and predicted quaternary structure. These groups were then analyzed in the context of biochemicallycharacterized bifurcating and non-bifurcating enzymes to demarcate their diverse functionalities.

Finally, we used this classification scheme to identify proteins that might be involved in the PTM of Hyds and to characterize their distribution in the Hyd classification groupings. The regulatory proteins identified include a histidine kinase-like $(\mathrm{Hkl})$ protein (sometimes annotated as HydE [21]), serine/threonine kinase (Stk), and serine phosphatase (Sp) that may be involved in PTM of putatively multimeric bifurcating Hyds. Herein we report a new classification scheme that assigns HydA into one of these three groups, based on the composition of proteins encoded in adjacent gene neighborhoods and structural features associated with these groups. Finally, we provide evidence from biochemical and mass spectrometry assays for PTM in multimeric Hyd complexes. 


\subsection{Identification and compilation of HydA homologs}

The catalytic subunit of [FeFe]-hydrogenase (HydA) from C. pasteurianum

(WP_004455619.1) was used in a BLASTp query to identify homologs present in publically

147 available genomes in the Department of Energy (DOE) Integrated Microbial Genomes (IMG)

148 database in June 2014 using default settings and an e-value of $10^{-5}$. The extracted homologs were 149 aligned using Clustal Omega [46] specifying default alignment parameters. Aligned homologs

150 were screened manually for the presence of three H-cluster binding motifs, previously defined as

151 L1 (TSCCPxW), L2 (MPCxxKxxE), and L3 (ExMACxxGCxxGGGxP) [34]. A total of 714

152 sequences were identified that contained the signature L1, L2, and L3 motifs. Despite previously 153 reported conservation of the three motifs [34], our analyses indicated slight variations in each of 154 them. Therefore, we analyzed the extent of variation in these three H-cluster binding motifs 155 using the weblogo server [47]. The taxonomy associated with each HydA sequence was 156 compiled using the DOE-IMG database.

\subsection{Construction of an extended sequence dataset}

A custom python (ver. 2.7.6) script was used to extract gene sequences (10 upstream and

16010 downstream) that flanked each hydA. The twenty inferred protein sequences were clustered by

161 pairwise BLASTp using the NCBI blastclust program [48] specifying a threshold of 30\%

162 sequence identities and a coverage of $60 \%$ for each pair of sequences. Clusters that contained a

163 minimum of 12 sequences were considered for further analysis, resulting in a total of 123 
164 clusters. The protein sequences within each of the 123 clusters were blasted against the CDD: a

165 public database that contains all known protein motifs and domains [49] using an e-value of 0.01

166 to confirm bin assignments that were made by cluster analysis. Protein sequences were pruned

167 from the clusters based on the absence of key domains (as identified by CDD blast) associated

168 with each protein cluster. In rare cases the proteins were manually reassigned to other clusters.

169 The filtered protein clusters were then annotated using the UniProt database [50]. Proteins

170 encoded in the gene neighborhood of hydA are reported in Supp. Table 1.

171

172

\subsection{Frequent pattern mining using the extended dataset}

The protein clusters $(n=123)$ encoded in the gene neighborhoods of the 714 HydA

174 homologs were converted into binary matrix for use in classifying the HydA homologs based on

175 the presence/absence of proteins. To objectively identify the most frequent patterns in the

176 composition of proteins encoded by genes flanking hydA, the Apriori algorithm was used as

177 implemented with the arules package in $\mathrm{R}$ [51]. Apriori is an algorithm that identifies frequent

178 items (i.e., protein clusters) within a database and characterizes associations among the

179 distribution of those items to objectively identify patterns. We applied the Apriori algorithm to

180 our protein cluster database while specifying the following parameters: a confidence threshold of

1810.95 and a support threshold of $0.1[51,52]$. The confidence threshold is the measure of the

182 statistical significance of an identified pattern within a dataset while the support threshold is

183 indicative of the relative abundance of a given pattern in a dataset. The Apriori algorithm works

184 in two phases with the first phase consisting of a scan of the entire database of proteins encoded

185 in flanking regions of hydA in order to objectively identify and extract frequently observed

186 proteins. In the second phase, the algorithm uses those frequently occurring proteins to identify 
187 the dominant patterns in the distribution of these proteins or their combinations. In doing so, the 188 algorithm identifies correlations (within the user defined confidence and support threshold) 189 between and among proteins in the database [52]. The 32 most frequent patterns and their 190 confidence intervals are reported in Supp. Table 2.

\subsection{Statistical analysis}

To further evaluate the extent to which Hyd group designations correspond to differences

194 in the composition of proteins encoded in the flanking regions of hydA, the binary

195 presence/absence table of the protein encoding genes flanking hydA (+/- 10 genes) were

196 subjected to Principal Component Analysis (PCA). Only proteins that were present in flanking

197 genes that were represented in $>2 \%$ of all HydA encoding genomes were considered in the 198 generation of the dissimilarity matrix. The dissimilarity matrix was subjected to PCA (using 199 variance-covariance matrix) to visualize variation in the composition of genes that flank hydA 200 using PAST (version 3) [53].

\subsection{Network analysis}

The abundant patterns that were (i.e. $\geq 95 \%$ confidence value and $\geq 10 \%$ support value)

204 identified by the Apriori algorithm were used to create a pattern correlating network with

205 Cytoscape specifying the force directed organic layout [54]. Each unique pattern was denoted as 206 a node and the edges between nodes represent the degree of correlation between the nodes. The 207 network was further analyzed using the Network Analyzer plugin in Cytoscape [55] to calculate 208 the relatedness (i.e., betweenness centrality) of each node in the network where the edges 
represent the degree of correlation between the nodes. Proteins with the highest betweenness centrality were overlaid on the network.

Proteins encoded in the flanking regions of hydA that had a relative frequency of $\geq 10 \%$ were extracted and used to create a binary matrix to identify the presence/absence of the protein in the gene context of each hydA. This matrix was then used to generate a network map to identify the protein clusters most commonly identified in each Hyd group. Cytoscape was used

to visualize the network using a force directed organic layout and the network was further analyzed using the Network Analyzer plugin.

\subsection{Determining the F- and C-cluster composition of HydA homologs}

The $\mathrm{N}$ - and C-terminal regions that flank the H-cluster (i.e., F- and C-cluster domains, respectively) of HydA were trimmed from alignment blocks and were subjected to BLASTp against CDD using the CDSEARCH/cdd v3.13 algorithm [49] (version 3.13) using an e-value of 0.01 as previously described [14]. The identified F- and C-cluster domains were compiled for each sequence using a custom python (ver. 2.7.6) script and were used to classify the putative domains of HydA and to determine if these structures varied according to group designation.

\subsection{Analysis of Post-Translational Modification}

The Hyd (i.e. HydABC) from T. maritima was purified as described previously [22] while purification and properties of the HydABCD enzyme from $C$. bescii will be reported elsewhere. Pyrococcus furiosus transhydrogenase (also known as NfnI or sulfide dehydrogenase) was purified as previously described [56]. 
PTM identification (phosphorylation) was conducted on intact protein complexes.

234 Samples were generated by spiking $4 \mu \mathrm{g}$ of protein complexes with Calf Intestinal Alkaline

235 Phosphatase (CIP, New England BioLabs) (1 CIP unit/1 $\mu \mathrm{g}$ protein) and incubation for $3 \mathrm{~h}$. at 37

$236{ }^{\circ} \mathrm{C}$ in $50 \mathrm{mM}$ ammonium bicarbonate, $\mathrm{pH}$ 8.5. Casein (Affymetrix Life Science) was chosen as a

237 positive control for phosphorylation. After treatment samples were immediately mixed with gel-

238 loading buffer, boiled for 5 min., centrifuged, and loaded onto 4-20\% mini-protean TGX gel

239 (Bio-Rad). Controls were prepared using the same protocol, however no CIP was added. Proteins 240 were visualized with Pro-Q Diamond Phosphoprotein Stain (Invitrogen/Molecular Probes) and 241 GelCode Blue Stain (ThermoFisher) according to manufacturer instructions.

244 (UPLC) series chromatography stack (Agilent Technologies) coupled directly to an electrospray245 time of flight (ESI-TOF) mass spectrometer (Bruker Micro-TOF). Before infusion to ESI source, 246 samples were separated on a reverse-phase (RP) column PLRP-S column (50 x $2.1 \mathrm{~mm}, 3 \mu \mathrm{m}$, $247100 \AA$ A , Agilent Technologies) or a size exclusion (SEC) Polyhydroxyethyl A column (100 x 4.6 $248 \mathrm{~mm}, 5 \mu \mathrm{m}, 500 \AA$ A, PolyLC Inc.) with flow rates of $600 \mu \mathrm{L} / \mathrm{min}$. (RP column) and $300 \mu \mathrm{L} / \mathrm{min}$. 249 (SEC column). Fast gradient conditions (1 min., 10\% B; 1.0-6.0 min., 10-70\% B; 6.0-7.0 min., $25010 \% \mathrm{~B}$ ) were applied to elute proteins from the RP column (temperature of $50^{\circ} \mathrm{C}$ ), while 251 isocratic conditions (25\% B) were used to elute proteins for $4 \mathrm{~min}$. from the SEC column 252 (temperature of $25^{\circ} \mathrm{C}$ ). Solvent A comprised $0.1 \%$ formic acid (FA, Sigma) in water 253 (ThermoFisher) and solvent B comprised 0.1\% FA in acetonitrile (ThermoFisher). 254 
2563.5 bar (RP and SEC columns, respectively), drying gas at a flow rate of 7.0 or $6.0 \mathrm{~L} / \mathrm{min}$. (RP

257 and SEC columns, respectively), drying temperature at $200^{\circ} \mathrm{C}$, capillary voltage at $4.5 \mathrm{kV}$, and

258 capillary exit voltage at $100 \mathrm{~V}$. Data was collected in positive mode only at $2 \mathrm{~Hz}$ rate over the

259 200-3000 m/z scan range. Data processing and analysis were performed using the Bruker Data

260 Analysis package 4.0. Charge deconvolution was performed using a maximum entropy algorithm

261 for $\mathrm{H}^{+}$adducts only and $0.1 \mathrm{~m} / \mathrm{z}$ data point spacing. The low mass end was defined by the mass

262 of lightest component while the high mass end was defined as 3.3x of the heaviest component

263 within the complex. Measured $\mathrm{m} / \mathrm{z}$ errors for all proteins were less than $0.8 \mathrm{~m} / \mathrm{z}$ from calculated

264 values.

265

266

3. RESULTS AND DISCUSSION

267

268

3.1 Taxonomic distribution and classification of HydA homologs.

The taxonomic distribution of hydA was examined in 30 bacterial, 5 archaeal, and 9

270 eukaryotic phyla with available and complete genome sequences, as determined in June 2014

271 (Table 1). None of the archaeal phyla (167 total genomes) contained organisms whose genomes

272 encoded for HydA while 17 of the 30 bacterial phyla (287 total genomes) contained organisms

273 whose genomes encoded for HydA. Three of the 9 eukaryotic phyla (5 total genomes) contained

274 organisms whose genomes encoded for HydA. Ninety percent of the 714 hydA homologs

275 identified were distributed among the bacterial phyla Firmicutes, Proteobacteria, Spirochaetes,

276 Thermotogae and which encoded on average $\sim 2$ HydA isoforms per HydA encoding genome. 
The genes flanking (+/- 10 genes) the 714 hydA homologs were compiled and the

279

280

281

282

283

284

285

286

287

288

289

290

291

292

293

294

295

296

297

298

299

300

encoded proteins clustered into homologous bins. The Apriori algorithm was then applied to this dataset to identify genes present at a high frequency in genomic regions flanking hydA and to calculate their co-occurrence patterns for use in predicting potential interactions. HydB and HydC were the most prevalent proteins encoded in genomic regions flanking hydA and these were identified in $\sim 40 \%$ of the 714 hydA flanking gene neighborhoods (Fig. 1). An ABC transporter protein, HydD, and Hkl were the next most frequently identified proteins which were encoded in $\sim 28 \%, 15 \%$, and $12 \%$ of the hydA flanking gene neighborhoods, respectively (Fig. 1).

The categorized proteins from the first phase of the Apriori algorithm were then used to generate association rules for classifying HydA based on the distribution of proteins or combinations therein encoded in the flanking regions of hydA. A total of 32 different flanking protein combinations were identified that were within the confidence value of 0.95 or higher (Supplementary Table 1). Of the 32 statistically supported flanking protein combinations, 98\% of them contained either HydB, HydC, HydD, or combinations of these proteins

(Supplementary Table 1). The identified flanking protein combinations $(n=32)$ were subjected to network analysis to identify the most prevalent co-occurrence patterns.

HydB, HydC, HydD, Hkl, a protein containing a polymerase and histidinol phosphatase (PHP) domain, and a protein with a DRTGG domain also had high betweenness centrality (e.g., connectivity among nodes) (Fig. 2). This indicates that these proteins were shared with a number of the 32 patterns and therefore behave as 'bridges' that connect the entire protein cluster 
301

302

303

304

305

network. Of these proteins, HydB, HydC, and HydD had the highest betweenness centrality. This indicates that these three proteins are highly represented in flanking regions of genomes that encode hydA and presumably, given the available biochemical evidence discussed below, interact with HydA.

To further characterize the proteins in the flanking regions of hydA, a network map was generated using the proteins that were identified within 10 genes upstream and downstream of $>2 \%$ of the 714 hydA homologs (Fig. 3). This network, like that presented in Fig. 2, again indicates that the $\mathrm{HydB}$ and $\mathrm{HydC}$ are highly prevalent in hydA gene neighborhoods and their distribution is strongly co-correlated. Likewise, the distribution of $\mathrm{HydD}$ and the Hkl protein also strongly co-vary. Rex (redox sensing repressor protein which is involved in transcriptional redox regulation [57-59]) was commonly found in the gene neighborhood of all three groups of Hyd (thus giving it a high 'betweenness centrality' value) and may represent a general transcriptional level mechanism to regulate Hyd as a function of cellular redox status. Based on these results we categorized each HydA into 3 groups: Group 1 (G1), Group 2 (G2), and Group 3 (G3), which are differentiated primarily on the basis of the presence of HydBCD (Fig. 4 and Supplementary

Table 2). The Hyds identified in each group were then compared to those of biochemically characterized Hyds [21, 35-37, 40,60] in order to putatively assign functionality and quaternary structure. while $\mathrm{HydC}$ is predicted to ligate a $[2 \mathrm{Fe}-2 \mathrm{~S}]$ cluster. Electron paramagnetic resonance (EPR) on 
324 HydB from T. maritima also indicated the presence of multiple iron-sulfur clusters and sequence

325 analysis of HydB predicted the presence of a FMN binding site and a variable number of iron-

326 sulfur clusters [22]. Similarly, bioinformatics analysis of HydB from A. woodii, C.

327 autoethanogenum, $M$. thermoacetica, and $R$. albus also indicated the presence of variable

328 number of iron sulfur clusters and a FMN binding site [21, 35-37, 40]. Sequence analysis of

329 HydC from T. maritima predicted the presence of one [2Fe-2S] cluster [22, 35]. In addition,

330 based on our sequence analysis, HydD exhibits homology to a thioredoxin-like Fd and, based on

331 the presence of two vicinal Cys residues, is predicted to bind a [2Fe-2S] cluster.

332

Hyd gene clusters that did not encode for or encoded incomplete HydB (e.g., truncated

HydB lacking a flavin binding site) and HydC proteins in the hydA gene neighborhood (i.e. +/-

10 genes) were classified as G1 Hyds ( $n=483$ HydA). Representatives of these monomeric

enzymes are from the bacterium $C$. pasteurianum $[2,11,14,16,34,61]$ and the alga $C$.

reinhardtii $[14,16,27,29,30]$. These have been subjected to extensive biochemical analysis and

are prototypical examples of this group. Biochemical data of HydA from C. pasteurianum [2, 11,

$33936]$ and $C$. reinhardtii $[27,29,30]$ indicate that they are unable to bifurcate electrons. These

340 findings suggest that the other G1-group Hyds also likely do not bifurcate, including the

341 previously characterized 'dimeric' Hyd from D. desulfuricans (note the presence of truncated

342 HydB which lacks a flavin binding site [23, 44]). Of the 483 G1 HydAs, the gene neighborhood

343 of seven homologs encoded for HydB, HydC, or HydD or a combination of these, but they did

344 not meet the classification scheme for complete HydBC in their gene neighborhoods. For

345 example, of these seven Hyds, one encoded for only HydB, three encoded for only HydC, one

346 encoded for only HydCD, and two encoded for only HydBD. Based on available biochemical 
347 data, it is not clear how or if these proteins interact with HydA. At this point, it is not known

348 whether these Hyds can bifurcate since all biochemically characterized bifurcating enzymes

349 encode for either HydABC or HydABCD complexes [21, 35-37, 40, 41].

350

351

352

353

354

355

356

357

358

359

360

361

362

363

364

365

366

367

Hyds that encode HydBC in the flanking regions of hydA were categorized as G2

enzymes ( $n=155$ HydA). Of these 155 Hyds, 92\% shared a common pattern of HydABC encoded in the ABC orientation (Fig. 4). Representatives of this Hyd group have been purified from $M$. thermoacetica and T. maritima, which were biochemically characterized as trimeric complexes (i.e. HydABC) $[35,36]$. In these examples, HydB is the subunit that has been predicted to contain a unique flavin binding site along with variable numbers of iron-sulfur clusters while HydC contains a single $[2 \mathrm{Fe}-2 \mathrm{~S}]$ cluster $[35,36]$. Both of these trimeric Hyds have been shown to bifurcate electrons, indicating that other G2 Hyds are likely capable of bifurcation $[35,36]$.

Both Fd and NADH are necessary for bifurcating Hyd activity and it has been proposed that HydC, which contains a Fd-like [2Fe-2S] cluster, accepts electrons from Fd while HydB accepts electrons from NADH [35]. If verified, this suggests the importance of both subunits HydBC for Hyd and bifurcating capability. Furthermore, the biochemical study conducted on the trimeric G2 Hyd from the thermophile T. maritima indicated that when HydBC were separated from HydA, the HydA enzyme lost its stability at elevated temperature and also lost the ability to reduce quinones [22]. This indicates that these two HydBC subunits not only play a role in electron transfer but also function to stabilize HydA.

In addition to HydBC, genes flanking certain G2 hydA were found to encode electron transfer proteins $(e t p)(\mathrm{n}=2)$, formate dehydrogenase $\mathrm{D}(f d h D)(\mathrm{n}=2)$, a homolog of HydA termed 
370 HydS (n=17), and Sp (n=28). HydS contains an additional sensory domain (i.e. PAS [45]) at its

371 C-terminus that is not present in canonical HydA. These results are consistent with previous

372 informatics studies which revealed the genes flanking hydA included hydS and $s p$ in $C$.

373 thermocellum DSM 1313 [37, 62] and T. maritima $[35,37]$. In addition, ETP and FdhD, the

374 latter of which has a NADP ${ }^{+}$binding site, have been shown to form a complex with G2 Hyds in

375 C. autoethanogenum [40]. This multimeric complex is involved in the reversible reduction of

$376 \mathrm{CO}_{2}$ in the presence of $\mathrm{H}_{2}$ to formate and also in the reversible reduction of Fd and $\mathrm{NADP}^{+}$using

377 formate. Interestingly, six of the $155 \mathrm{G} 2 \mathrm{Hyds}$ were found to have fused HydB and HydC

378 subunits and one G2 Hyd (Nyctotherus ovalis; AAU14235) was found to have fused HydABC

379 subunits. These observations provide additional evidence that these two proteins function

380 synergistically, as has been observed in numerous other co-occurring enzyme systems, including

381 Hyd maturases [1, 63-67].

382

Lastly, hydA-containing gene clusters that encode for HydBCD were classified into G3 enzymes (n=76). Interestingly, $71 \%$ of the G3 Hyds (58 of 76) also encoded an Hkl protein that

385 was present within the hyd gene cluster. The identity of Hkl proteins was confirmed based on the 386 presence of a HATPase_c motif (PF02518) which is the ATP binding site of the ATPase domain $387[68,69]$. This motif composition differs from canonical histidine kinase proteins. Canonical 388 histidine kinase typically has an additional two motifs including the HAMP motif [histidine 389 kinase, adenylyl cyclase, methyl-accepting protein and phosphatase (PF00672)] which is a 390 phosphoacceptor domain that acts as a cytoplasmic intermediate linker connecting the 391 phosphoryl transfer (HisKA) motif [68, 70-74] with the sensor domains outside of cytoplasm $392[70,75,76]$. Biochemical characterization of the G3 Hyd in A. woodii indicates that it forms a 
tetrameric complex that includes HydBC and a Fd-like HydD protein that contains a [2Fe-2S] cluster [21].

$$
\text { been shown to bifurcate and also to require two electron carriers, Fd and NADH, for activity }
$$
[21]. However, unlike the trimeric G2 Hyd identified in T. maritima [35], which is involved in fermentative $\mathrm{H}_{2}$ production, the tetrameric G3 Hyd identified in A. woodii is involved in oxidation of $\mathrm{H}_{2}$ [35]. It has been proposed that electrons are transferred from HydA to HydC and then to HydB where the electrons are bifurcated simultaneously to reduce Fd and $\mathrm{NAD}^{+}[21]$. This mechanism, like that proposed for trimeric G2 Hyd [35], implies an important role for HydBC in electron transfer. The function of HydD in G3 Hyd and its role in bifurcation and in reaction directionality is still unknown. Importantly, in several instances, HydBCD were not present in this arrangement, but rather, were interspersed among other genes in the 20 protein encoding genes flanking G3 hydA. Examples include Hyd from Desulfarculus baarsii DSM 2075 (Deba_1523), Desulfobacterium autotrophicum HRM2 (HRM2_16550), and Kosmotoga olearia TBF 19.5.1 (Kole_0172). Thus, it is unclear if these Hyds have the capability to bifurcate. In addition to HydBCD and Hkl, genes flanking a number of G3 Hyds encoded various regulatory genes including Stk proteins $(n=39 \mathrm{HydA})$, and/or transpeptidase proteins $(\mathrm{n}=20 \mathrm{HydA})$. In support of the group designations identified by Apriori algorithm classification, PCA ordination of a matrix describing the dissimilarity in the composition of proteins encoded by genes in flanking regions of hydA formed clusters that largely corresponded to the same group designations (Supplementary Figure. 1). A network map was also used to confirm the extent to 
416 which proteins encoded by genes in flanking regions of hydA correlated with group designation

417 (Fig. 5). Based on the Apriori analysis, regulatory genes including Sp and HydS were enriched in

418 G2 Hyds while Hkl, DRTGG, a PHP domain-containing protein and Stk were enriched in

419 flanking regions of G3 Hyds. The difference in regulatory gene association between the two

420 groups indicates that G2 and G3 Hyds may be subject to different modes of PTM and/or that

421 these PTM enzymes exhibit specificity for target Hyd proteins allowing for their differential

422 regulation if both types of protein are present in the same organism.

423

424

The HydA copy number per genome varied as a function of taxonomy (Table 1).

425 Moreover, the HydA copy number per genome, when broken out by group designation, also

426 varied (Supplementary Figure 2A). Multiple copies (isoforms) of G1 Hyd in the same genome

427 was the most common pattern identified ( $n=117$ of 287 genomes). Several genomes contained

428 three or more copies of HydA, with a maximum copy number/genome of seven HydAs

429 (Desulfotomaculum carboxydivorans CO-1-SRB, DSM 14880). Fifty-two percent of the HydA-

430 encoding genomes encoded for at least two different types of Hyd (Supplementary Figure 2B).

431 For example, 77 genomes encoded for G1 and G2 Hyds, 44 encoded for G2 and G3 Hyds, and

43217 genomes encoded for all three groups of Hyds. The co-existence of G2 and G3 Hyds with G1

433 Hyds potentially suggests they have different functional roles within the organisms and again is

434 consistent with data presented above indicating that they may be regulated at the post-

435 translational level by different PTM enzymes.

436

437

Hyd variants were distributed unequally among bacterial phylum-level lineages. G1 Hyds

438 were prevalent in the Bacteroidetes, Firmicutes, Proteobacteria, Spirochaetes and Thermotogae 
phyla while G2 Hyds were enriched in Choloroflexi and Thermotoga phyla. G3 Hyds were enriched in Firmicutes and Spirochaetes phyla (Table 1). Two of the three eukaryotic phyla that contained homologs of Hyd encoded G1 Hyds: heterokonts with one genome (i.e. Thalassiosira pseudonana) and chlorophytes with 3 genomes. One of the three eukaryotic phyla encoded a G2 Hyd: Ciliophora with one genome (i.e. Nyctotherus ovalis). Multiple copies of HydA were often encoded in chlorophyte genomes and these were all classified as G1 Hyd. Interestingly, HydA identified in the ciliate Nyctotherus ovalis [77-79] forms a fused complex with HydB-like and HydC-like subunits, which begs the question as to whether this enzyme can also bifurcate electrons as it meets the minimal requirement of gene complements (i.e., encodes HydABC) hypothesized to afford bifurcation activity.

\subsection{Structural composition of HydA (H-, F- and C- cluster).}

Various biochemical $[2,11,29,30]$ and bioinformatics studies [14, 16, 32, 34, 61] indicate substantial variation in the iron-sulfur cluster content of the F- and C- clusters of HydA, which are hypothesized to act as electron transfer conduits for the various Hyds. Thus, the composition of iron-sulfur cluster binding domains in the F- and C-clusters of HydA were characterized in order to determine if variations in these clusters correlated with Hyd group designations. Based on the presence or absence of cluster binding motifs and other identifiable motifs defined in the CDD, the 714 HydA homologs were categorized into modular structures according to the scheme of Calusinska et al., 2010 [14]. A total of 12 modular structures were identified and these were nearly equally represented across all groups of Hyd (Fig. 6). Out of 12 modular structures, 10 were predicted to ligate two [4Fe-4S] clusters in the F-cluster domain. More than 50\% of HydA in G2 and G3 exhibited the M2i modular structure which were 
462 predicted to ligate two [4Fe-4S] clusters or the M3a modular structure which comprised multiple 463 iron-sulfur clusters (i.e. [2Fe-2S], histidine substituted [4Fe-4S] and 2[4Fe-4S]) in the F-cluster

464 domain. Likewise, more than $50 \%$ of the HydA lacked C-clusters and of those that did have C465 clusters, most were classified as the non-bifurcating G1 Hyds.

We also examined the variation in the three amino acid sequence motifs (i.e. L1, L2 and L3 $[14,16])$ that are involved in ligating the H-cluster in relation to group designations (Fig. 7).

469 The majority of the alignment positions were comprised of residues that did not vary

470 substantially in amino acid usage for any single group. However, two positions in each of the

471 three motifs contained residues that differed among groups. For example, in the L1 motif region, 472 the $4^{\text {th }}$ residue, cysteine, is more common in G1 and G2 Hyds while serine is more common in

473 G3 Hyds. Similarly, glycine is commonly observed in the $6^{\text {th }}$ position of the L1 motif in G2 and 474 G3 Hyds, whereas alanine is more commonly observed at this position in G1 Hyds. Within the 475 L2 motif region, methionine is more common in the $1^{\text {st }}$ position in G2 and G3 Hyds, whereas 476 glycine is more common in G1 Hyds. Within the L3 motif region, glycine and valine in the $2^{\text {nd }}$

477 position are more common in G1 Hyds while valine and isoleucine are more common in G2 and 478 G3 Hyds. Finally, proline and glycine are more common in the $13^{\text {th }}$ position of the L3 motif in 479 G1 Hyds whereas glycine is more common in G2 and G3 Hyds. indicates that bifurcation capability is more likely to be predicated by the presence/absence of 483 accessory subunits, in particular the flavin binding subunit HydB. Moreover, these results may 484 suggest that variations in F-, C-, and H-cluster domains are refinements to enzymes that are 
485 primarily under selection for bifurcating versus non-bifurcating abilities. Biochemical

486 characterization of Hyds from A. woodii, C. autoethanogenum, M. thermoacetica and T.

487 maritima all showed the ability to bifurcate and all the Hyds identified in these species were

488 either trimeric or tetrameric [21, 35, 36, 40]. Conversely, the monomeric Hyd from $C$.

489 pasteurianum $[2,11,36]$ and $C$. reinhardtii $[27,29,30]$ indicate that they are unable to bifurcate

490 electrons. These findings suggest that the other G1-group Hyds also likely do not bifurcate,

491 including the 'dimeric' Hyd from $D$. desulfuricans which forms a complex with a truncated

492 HydB that lacks a flavin binding site [23, 44]. A few additional putative dimeric forms of Hyd

493 were identified that contained full length $\mathrm{HydB}$ with a flavin binding motif, but due to lack of

494 biochemical characterization of an enzyme with this configuration, they were classified as G1

495 enzymes. For example, the HydA encoded in the Coprococcus catus GD/7 genome (locus tag:

496 CC1_10640) also encoded for HydB in its flanking region but lacked genes encoding for HydC.

497 Hence, based on available biochemical data, we hypothesize that the enzymes that lack either of

498 the subunits i.e. HydB or HydC do not have the capability to bifurcate.

\subsection{Post translational modification of bifurcating G2 and G3 Hyds}

Among the more intriguing and novel findings from our bioinformatics work was the

502 identification of genes encoding proteins that could be involved in PTM in the gene

503 neighborhoods of G2 and G3 Hyds. Identified proteins included Hkl and Stk in G3 Hyds while

504 Sp was commonly observed in G2 Hyds. Despite both having an ATPase domain and exhibiting $505 \sim 25 \%$ homology with $\sim 40 \%$ pairwise sequence coverage, an alignment of our identified Hkl and

506 Stk proteins indicated they are distinct (Supplementary Figure 3). Protein kinases have been a

507 subject of extensive study which have demonstrated their involvement in various regulatory 
processes. Among those most widely studied are Hkl and Stk which have been biochemically shown to target histidine $(\mathrm{H})$ and serine/threonine $(\mathrm{S} / \mathrm{T})$ for phosphorylation, respectively [70, 80-83]. The presence of $\mathrm{Hkl}$ and Stk in the gene neighborhood of G3 hydA indicate that these kinases and phosphatases may be involved in reversible phosphorylation of $\mathrm{H}, \mathrm{S}$ and $\mathrm{T}$ residues. By extension, this may suggest conservation of these residues in Hyd subunits.

Multiple sequence alignments (MSA) of all the sequences for the Hyd subunits, i.e. HydA, HydB, and HydC from G1, G2, and G3, revealed few or no conserved (i.e. $\geq 90 \%$ conservation) $\mathrm{H}, \mathrm{S}$, and T residues. We therefore screened alignments of only HydA and its subunits in gene clusters that also encode Sp (G2), Stk (G3), and Hkl (G3) in the flanking region, which resulted in a total of 18 and 46 sequences, respectively. When HydABC that were not flanked by Hkl or Stk were removed from MSA, several conserved H, S, and T were identified (Supplementary Figure 4, 5, and 6). HydB subunits also harbored conserved (i.e. $\geq 90 \%$ conservation) $\mathrm{H}$ and S/T residues regardless of whether they were classified as G2 or G3 Hyds (Supplementary Figure 5). HydC in G2 harbored multiple conserved (i.e. $\geq 90 \%$ conservation) $\mathrm{H}$ and $\mathrm{T}$ residues while $\mathrm{G} 3$ harbored multiple conserved $\mathrm{T}$ residues only at the same position (Supplementary Figure 6). Lastly, HydD in G3 also harbored conserved (i.e. $\geq 90 \%$ ) H and T residues (Supplemental Figures 7). However, the extent of conservation of H, S, and T residues in HydD did not vary substantially in enzymes that were not flanked by Hkl or Stk versus those that were flanked by these proteins. While the presence of conserved $\mathrm{H}$ and $\mathrm{S} / \mathrm{T}$ residues does not, by itself, indicate that those conserved sites are post-translationally modified, they do provide potential targets for in vitro studies. To provide further evidence for PTM of Hyd, we 
530 undertook a biochemical assay to probe PTM using the G2 Hyd from T. maritima and the G3 Hyd from Caldicellulosiruptor bescii.

Sequence analysis of the G2 HydA from T. maritima indicates that it comprises multiple 534 iron-sulfur cluster (i.e. 2[Fe-2S], histidine substituted [4Fe-4S] and 2[4Fe-4S]) binding motifs in 535 the F-cluster and a [2Fe-2S] cluster binding motif in the C-cluster and thus is classified within 536 the 3c modular structure (Fig. 6). The structural proteins identified in T. maritima G2 Hyd (i.e. 537 HydABC) and a homolog of Sp (TM1423) have been identified in the flanking region of the 538 trimeric Hyd complex (TM1424-TM1426). Similarly, sequence analysis of HydA from C. bescii 539 indicates that it also contains multiple iron-sulfur cluster (i.e. 2[Fe-2S], histidine substituted $540[4 \mathrm{Fe}-4 \mathrm{~S}]$ and $2[4 \mathrm{Fe}-4 \mathrm{~S}]$ ) binding motifs in its F-cluster and is thus classified as having the M3a 541 modular structure, which is a common structure associated with G3 Hyds (Fig. 6). The structural 542 proteins identified in C. bescii G3 Hyd (i.e. HydABCD) are encoded in an apparent operon 543 (YP_002573168 to YP_002573172). Homologs of Hkl (YP_002573169) and Stk

544 (YP_002573164) were also identified in the gene neighborhood of hydA in C. bescii. The 545 presence of $\mathrm{Hkl}$ and Stk in the flanking gene neighborhood of hydA in C. bescii and Sp in the 546 flanking region of hydA in T. maritima suggests that phosphorylation of these Hyd proteins may 547 occur.

To test the possibility for PTM, purified Hyd protein complexes from T. maritima and $C$. 550 bescii were analyzed by SDS-PAGE followed by phospho-specific protein staining. Image 551 analysis showed strong signals for HydA/B proteins (68/72 kDa for T. maritima and 63/64 kDa 552 for $C$. bescii) as well as for $\mathrm{HydD}(14 \mathrm{kDa})$ from $C$. bescii (Fig. 8, left panel). Positive (casein, 
553 Cas from Bos taurus) and negative (transhydrogenase, Nfn1 from Pyrococcus furiosus) controls

554 for phospho-staining were included on the gel for reference. To further establish the presence of

555 phosphorylation, protein samples were treated with calf intestinal phosphatase (CIP). A

556 reduction in phosphorylation intensity was observed for high molecular weight Hyd subunits

557 from T. maritima and casein. Incubation of C. bescii Hyd complex with CIP did not lead to a

558 significant change in fluorescence. The same gel was then stained with Coomassie Blue to verify

559 protein loading (Fig. 8, right panel). T. maritima HydC (18 kDa) was poorly visualized, as

560 observed by the less than stoichiometric band intensity. Coomassie stain showed that $\mathrm{HydC}$ (17

$561 \mathrm{kDa})$ and $\mathrm{HydD}(14 \mathrm{kDa})$ from $C$. bescii were present at an equal ratio, as expected, in contrast

562 to their signal when visualized for phosphorylation.

563

564

Mass spectrometry was used to further investigate Hyd subunit modification. High-

565 resolution mass analysis of intact proteins is a sensitive method for observing heterogeneity.

566 Purified Hyd complexes were subjected to size-exclusion and reverse-phase chromatography to

567 remove salts and buffers prior to electrospray time of flight mass analysis. All Hyd subunits,

568 except for HydC from C. bescii, were observed as a mixture of several predominant forms.

569 HydB from T. maritima showed significant heterogeneity, with all of the forms being 300-400

570 Da greater than the expected molecular weight of $68,551 \mathrm{Da}$, based on the amino acid sequence

571 (Fig. 9, top panel). HydC from T. maritima had three distinct forms separated by $98 \mathrm{Da}$ (Fig. 9,

572 middle panel). Analysis of $C$. bescii proteins showed that HydD was present as two dominant

573 forms (1:3 ratio), separated by $80 \mathrm{Da}$ (Fig. 9, bottom panel).

574 
Phosphorylation is often difficult to confirm by mass spectrometry due to sub-

576 stoichiometric modification and loss of the attached phosphate during sample processing and

577 electrospray ionization. Attachment of a phosphate group $\left(\mathrm{HPO}_{3}\right)$ adds $80 \mathrm{Da}$ to the protein for

578 each phosphorylated amino acid [84-87]) as we observed for HydD from $C$. bescii. In contrast, a

579 difference $98 \mathrm{Da}\left(\mathrm{H}_{3} \mathrm{PO}_{4}\right)$ is indicative of an ionization induced loss of phosphorylation specific

580 to serine and threonine residues $[86,88,89]$. This result suggests modification of serine and

581 threonine residues of T. maritima HydC, although phosphorylation at the peptide level will be

582 necessary to confirm the specific sites of phosphorylation. Together, the SDS-PAGE and LCMS

583 analysis strongly suggest that HydAB and HydC of T. maritima G2 Hyd are phosphorylated.

584 Along with our bioinformatics-based analysis, this provides strong evidence that Hyd proteins

585 are likely to be post-translationally modified. This is an attractive mechanism for regulating the

586 activity of bifurcating G2 Hyd complexes. Similarly, SDS-PAGE and LCMS analysis strongly

587 suggest that the HydAB and HydD subunits of $C$. bescii G3 Hyd are phosphorylated.

588 Interestingly, HydC in C. bescii G3 Hyd was not modified. Since G3 HydC lacked conserved H

589 residues but G3 HydABD all contained a number of conserved $\mathrm{H}$ residues, this result suggests

590 that $\mathrm{Hkl}$ is potentially responsible for phosphorylating the $\mathrm{H}$ residues in G3 HydA. Future

591 biochemical studies are needed to identify the specific sites that are putatively involved in

592 phosphorylation activity, their effects on modulating hydrogenase activities, and the specific

593 enzymes involved in PTM activities.

594

595

\section{CONCLUSIONS}


Our bioinformatics-based analysis of the gene neighborhood of hydA revealed three

major groups of Hyd (G1, G2, and G3) that are differentiated on the basis of the presence of HydBC (G2) or HydBCD (G3). The G1 enzymes appear to be monomeric and based on biochemical data are predicted to not bifurcate while G2 and G3 Hyds are in trimeric and tetrameric forms, respectively. Based on bioinformatics analysis and albeit limited biochemical data, G2 and G3 enzymes are predicted to bifurcate. Variation in the F- and C-clusters and the residues that ligate the $\mathrm{H}$-cluster of HydA do not vary in a systematic manner with respect to Hyd group designations, indicating that they are under different selection and unlikely to delineate the ability of enzymes to bifurcate electrons.

Our analysis also identified regulatory genes in the flanking regions of hydA, including Sp (in G2 Hyds), Stk (in G3 Hyds), and an Hkl (in G3 Hyds), all of which we hypothesize to play a role in PTM regulation of Hyds. Indeed, our MS and biochemical data indicate that examples of G2 and G3 Hyds are phosphorylated alluding to the role of the aforementioned proteins in potentially regulating Hyd. That PTM proteins associate with G2/G3 Hyd but not G1 Hyd is consistent with the prevalence of G1 Hyd in genomes that encode for G2/G3 Hyd, necessitating mechanisms to differentially regulate the activities of these enzymes. Moreover, the presence of different PTM proteins in the gene neighborhoods of G2 and G3 Hyds and differences in the protein subunits that were apparently phosphorylated is consistent with the cooccurrence of G2 and G3 Hyd in the same organisms and their need to differentially regulate these enzyme complexes. Collectively, these results show that the vast inferred structural diversity of Hyds can be classified into only three fundamental groups, and variations within them allude to their various functions in microbial metabolism. The classification scheme 
620 reported herein provides the first framework for prioritizing biochemical and mutagenesis studies

621 of Hyds aimed at furthering our understanding of the functional role of this surprisingly cohesive 622 group of enzymes.

623

624

\section{ACKNOWLEDGEMENTS}

625 This work was supported as part of the Biological Electron Transfer and Catalysis Energy

626 Frontier Research Center funded by the U.S. Department of Energy, Office of Science, and Basic

627 Energy Sciences under Award \# DE-SC0012518.

628

629

\section{FIGURE LEGENDS}

630

631

Figure 1. Relative frequency of encoded proteins that were identified among genes flanking 714

632 hydA homologs (+/-10 genes). For simplicity, only those proteins that were encoded in $\geq 6 \%$ of

633 the 714 gene neighborhoods are shown. The identity of all proteins that flank each individual

634 HydA can be found in Supplementary Table 2. (Single column).

635

636 Figure 2. Network map depicting the dominant patterns in proteins encoded in the genes that

637 flank hydA (+/- 10 genes), as identified by the Apriori algorithm (18). Each unique pattern is

638 given as a node and the edges between nodes represent significant correlations between those

639 nodes (confidence value of $\geq 0.95$ ). Node color represents the betweenness centrality (a measure

640 of the 'connectedness' of each gene to the total network) that connects multiple groups (i.e., are

641 shared in multiple groups). Node size represents the number of patterns/genes significantly

642 associated with other patterns/genes. The force directed organic layout within Cytoscape was 
643 used to visualize the correlation in the network. Patterns in the distribution of genes flanking

$644 h y d A$, as revealed by this network, were used to classify the 714 HydA homologs into 3

645 categories (described in text). For simplicity, only nodes with betweenness centrality of 0.06 or

646 greater are labeled. (Single column)

647

648 Figure 3. Network map depicting the correlation of proteins encoded by genes flanking $(+/-10$ 649 genes) hydA. Each unique protein is given as a node and the edges between nodes represent 650 significantly correlated proteins $(p<0.05)$. Node color represents the betweenness centrality that 651 connects proteins (a measure of the 'connectedness' of each gene to the total network) while 652 edge color represents the Pearson correlation describing the co-distribution of proteins encoded 653 in flanking regions of hydA. Node size represents the number of proteins encoded by genes 654 flanking hydA that exhibit significant associations with other proteins in these flanking regions. 655 (Double column)

656

657 Figure 4. Classification of HydA ( $n=714)$ homologs based on the composition of proteins 658 encoded in flanking gene neighborhoods (+/-10 genes). The number of HydA homologs that fall 659 within each group is indicated in parentheses. The percent of HydA homologs within a specified 660 group that exhibit the exact gene neighborhood as depicted is given as a percentage. An 661 organism and the locus tag for a representative gene cluster for each specified group is indicated.

662 Asterisks denote Hyd homologs that have been biochemically characterized (see results and 663 discussion for details). Those with a red asterisk have been biochemically shown to bifurcate 664 while those with a black asterisk have been biochemically shown to not bifurcate. (Double 665 Column) 
667 Figure 5. Network analysis of proteins encoded by genes flanking (+/-10 genes) hydA. Only

668 proteins $(n=22)$ encoded in the flanking regions of $>10 \%$ of the each HydA group (i.e. relative

669 frequency of $>10 \%$ ) were considered in this analysis. Here, node color represents the

670 betweenness centrality (a measure of the 'connectedness' of each gene to the total network)

671 while edge color represents the relative frequency of each protein in each group. The force

672 directed organic layout was used to visualize the correlation in the network. The average pattern

673 associated with each of the 3 defined Hyd groups were included in this analysis [indicated by

674 Group 1 (G1), Group 2 (G2), and Group 3 (G3)]. (Single column)

675

676 Figure 6. Inferred domain composition of the F- and C-clusters associated with HydA homologs.

677 Homologs and modular structures are organized by Hyd group designations given on the left side

678 of the figure. The number in the parenthesis next to the modular structure designation represents 679 the number of HydA homologs out of 714 that have the specified modular structure. An example 680 of a HydA in an organism and its associated locus tag with each modular structure is indicated 681 on the right side of the figure. Hyd homologs that have been biochemically characterized are 682 denoted with an asterisk. Hyd homologs with a red asterisk have been biochemically shown to 683 bifurcate while those with a black asterisk have been biochemically shown to not bifurcate. 684 (Double column)

686 Figure 7. Schematic diagram indicating the extent of conservation in residues comprising the H687 cluster motifs (L1, L2, and L3, as outlined by [11]) in HydA as organized by Hyd group 
688 designations. Residues with higher bit scores (i.e., larger font sizes) suggest a greater degree of 689 conservation at a given aligned position within a given group. (Single column)

690

691 Figure 8. Phosphoprotein-specific staining. Purified Hyd were separated using SDS-PAGE and 692 stained with a fluorescent dye that is specific for phosphoproteins (left panel) followed by 693 Coomassie staining (right panel) to observe protein abundance. Samples were run with and 694 without treatment with calf intestinal phosphatase (CIP). The G2 Hyd from Thermotoga 695 maritima $(\mathrm{Tm})$ and the G3 Hyd from Caldicellulosiruptor bescii $(\mathrm{Cb})$ were positive for 696 phosphorylation. Casein (Cas) from Bos taurus and transhydrogenase (Nfn1) from Pyrococcus 697 furiosus served as positive and negative controls for phosphorylation, respectively. (Single 698 column)

699

700 Figure 9. LCMS analysis of Hyd subunits from T. maritima (Tm) and C. bescii (Cb). Intact 701 protein analysis of G2 and G3 Hyd separated by SEC-ESI-Q-TOF shows heterogeneity of 702 proteins. The $\operatorname{HydB}(\mathrm{G} 2)$ and $\operatorname{HydC}(\mathrm{G} 2)$ subunits have three prominent forms, expected $\mathrm{MW}=$ $70368,551 \mathrm{Da}$ (top panel) and expected MW = 18,193 Da (middle panel), respectively. The HydD 704 (G3) subunit (expected MW = 14,688 Da, bottom panel) was found in two major forms spaced 705 by $80 \mathrm{Da}$. (Single column)

706

707

708

709

710 


\section{REFERENCES}

712

713 [1] D.W. Mulder, E.S. Boyd, R. Sarma, R.K. Lange, J.A. Endrizzi, J.B. Broderick, J.W. Peters,

714 Stepwise $[\mathrm{FeFe}]$-hydrogenase H-cluster assembly revealed in the structure of $\mathrm{HydA}^{\Delta \mathrm{EFG}}$, Nature, $715465(2010) 248-251$.

716 [2] J.W. Peters, W.N. Lanzilotta, B.J. Lemon, L.C. Seefeldt, X-ray crystal structure of the Fe717 only hydrogenase $(\mathrm{Cpl})$ from Clostridium pasteurianum to 1.8 angstrom resolution, Science, 282 718 (1998) 1853-1858.

719 [3] J.A. Stapleton, J.R. Swartz, A cell-free microtiter plate screen for improved [FeFe]720 hydrogenases, PLOS ONE, (2010).

721 [4] J.A. Stapleton, J.R. Swartz, Development of an in vitro compartmentalization screen for 722 high-throughput directed evolution of [FeFe]-hydrogenases, PLOS ONE, (2010).

723 [5] R. Cammack, Michel F, and Robert R., Hydrogen as a Fuel: Learning from Nature, Taylor 724 and Francis Inc., New York, USA, 2001.

725 [6] W. Lubitz, H. Ogata, O. Rudiger, E. Reijerse, Hydrogenases, Chemical Reviews, 114 (2014) $726 \quad 4081-4148$.

727 [7] T.M. VanderSpek, A.F. Arendsen, R.P. Happe, S.Y. Yun, K.A. Bagley, D.J. Stufkens, W.R. 728 Hagen, S.P.J. Albracht, Similarities in the architecture of the active sites of Ni-hydrogenases and 729 Fe-hydrogenases detected by means of infrared spectroscopy, European Journal of Biochemistry, $730237(1996) 629-634$.

731 [8] S. Shima, R.K. Thauer, U. Ermler, Carbon monoxide as intrinsic ligand to iron in the active 732 site of [Fe]-hydrogenase, Metal ions in life sciences, 6 (2009) 219-240. 
733 [9] S. Shima, O. Pilak, S. Vogt, M. Schick, M.S. Stagni, W. Meyer-Klaucke, E. Warkentin, R.K.

734 Thauer, U. Ermler, The crystal structure of [Fe]-hydrogenase reveals the geometry of the active 735 site, Science, 321 (2008) 572-575.

736 [10] S. Shima, R.K. Thauer, A third type of hydrogenase catalyzing $\mathrm{H}_{2}$ activation, The Chemical 737 Record, 7 (2007) 37-46.

738 [11] M.W.W. Adams, The structure and mechanism of iron-hydrogenases, Biochimica Et 739 Biophysica Acta-Bioenergetics, 1020 (1990) 115-145.

740 [12] R. Cammack, Bioinorganic chemistry - Hydrogenase sophistication, Nature, 397 (1999) $741 \quad 214-215$.

742 [13] K.A. Vincent, A. Parkin, F.A. Armstrong, Investigating and exploiting the electrocatalytic 743 properties of hydrogenases, Chemical Reviews, 107 (2007) 4366-4413.

744 [14] M. Calusinska, T. Happe, B. Joris, A. Wilmotte, The surprising diversity of clostridial 745 hydrogenases: a comparative genomic perspective, Microbiology, 156 (2010) 1575-1588.

746 [15] D.S. Horner, B. Heil, T. Happe, T.M. Embley, Iron hydrogenases - ancient enzymes in 747 modern eukaryotes, Trends in Biochemical Sciences, 27 (2002) 148-153.

748 [16] J. Meyer, [FeFe] hydrogenases and their evolution: a genomic perspective, Cellular and 749 Molecular Life Sciences, 64 (2007) 1063-1084.

750 [17] J.W. Peters, G.J. Schut, E.S. Boyd, D.W. Mulder, E.M. Shepard, J.B. Broderick, P.W. King, 751 M.W.W. Adams, FeFe - and NiFe -hydrogenase diversity, mechanism, and maturation, 752 Biochimica Et Biophysica Acta-Molecular Cell Research, 1853 (2015) 1350-1369.

753 [18] S.A. Caffrey, H.S. Park, J.K. Voordouw, Z. He, J. Zhou, G. Voordouw, Function of 754 periplasmic hydrogenases in the sulfate-reducing bacterium Desulfovibrio vulgaris 755 Hildenborough, Journal of Bacteriology, 189 (2007) 6159-6167. 
756 [19] J.M. Odom, H.D. Peck, Hydrogen cycling as a general mechanism for energy coupling in 757 the sulfate-reducing bacteria, Desulfovibrio sp., Fems Microbiology Letters, 12 (1981) 47-50.

758 [20] J.M. Odom, H.D. Peck, Hydrogenase, electron-transfer proteins, and energy coupling in the 759 sulfate-reducing bacteria Desulfovibrio, Annual Review of Microbiology, 38 (1984) 551-592.

760 [21] K. Schuchmann, V. Muller, A bacterial electron-bifurcating hydrogenase, Journal of 761 Biological Chemistry, 287 (2012) 31165-31171.

762 [22] M. Verhagen, T. O'Rourke, M.W.W. Adams, The hyperthermophilic bacterium,

763 Thermotoga maritima, contains an unusually complex iron-hydrogenase: amino acid sequence 764 analyses versus biochemical characterization, Biochimica Et Biophysica Acta-Bioenergetics, $7651412(1999)$ 212-229.

766 [23] Y. Nicolet, C. Piras, P. Legrand, C.E. Hatchikian, J.C. Fontecilla-Camps, Desulfovibrio 767 desulfuricans iron hydrogenase: the structure shows unusual coordination to an active site $\mathrm{Fe}$ 768 binuclear center, Structure, 7 (1999) 13-23.

769 [24] G. Voordouw, V. Niviere, F.G. Ferris, P.M. Fedorak, D.W.S. Westlake, Distribution of 770 hydrogenase genes in Desulfovibrio spp. and their use in identification of species from the oil 771 field environment, Applied and Environmental Microbiology, 56 (1990) 3748-3754.

772 [25] Y. Nicolet, B.J. Lemon, J.C. Fontecilla-Camps, J.W. Peters, A novel FeS cluster in Fe-only 773 hydrogenases, Trends in Biochemical Sciences, 25 (2000) 138-143.

774 [26] E.S. Boyd, T.L. Hamilton, K.D. Swanson, A.E. Howells, B.K. Baxter, J.E. Meuser, M.C. 775 Posewitz, J.W. Peters, [FeFe]-hydrogenase abundance and diversity along a vertical redox 776 gradient in Great Salt Lake, USA, International Journal of Molecular Sciences, 15 (2014) 2194777721966. 
778 [27] M. Forestier, P. King, L.P. Zhang, M. Posewitz, S. Schwarzer, T. Happe, M.L. Ghirardi, M.

779 Seibert, Expression of two [Fe]-hydrogenases in Chlamydomonas reinhardtii under anaerobic

780 conditions, European Journal of Biochemistry, 270 (2003) 2750-2758.

781 [28] M.L. Ghirardi, M.C. Posewitz, P.C. Maness, A. Dubini, J.P. Yu, M. Seibert, Hydrogenases

782 and hydrogen photoproduction in oxygenic photosynthetic organisms, Annual Review of Plant

783 Biology, 58 (2007) 71-91.

784 [29] T. Happe, A. Kaminski, Differential regulation of the Fe-hydrogenase during anaerobic

785 adaptation in the green alga Chlamydomonas reinhardtii, European Journal of Biochemistry, 269

786 (2002) 1022-1032.

787 [30] T. Happe, B. Mosler, J.D. Naber, Induction, localization and metal content of hydrogenase

788 in the green alga Chlamydomonas reinhardtii, European Journal of Biochemistry, 222 (1994)

$789 \quad 769-774$.

790 [31] D.W. Mulder, E.M. Shepard, J.E. Meuser, N. Joshi, P.W. King, M.C. Posewitz, J.B.

791 Broderick, J.W. Peters, Insights into [FeFe]-hydrogenase structure, mechanism, and maturation,

792 Structure, 19 (2011) 1038-1052.

793 [32] J.E. Meuser, E.S. Boyd, G. Ananyev, D. Karns, R. Radakovits, U.M.N. Murthy, M.L.

794 Ghirardi, G.C. Dismukes, J.W. Peters, M.C. Posewitz, Evolutionary significance of an algal gene

795 encoding an [FeFe]-hydrogenase with F-domain homology and hydrogenase activity in Chlorella

796 variabilis NC64A, Planta, 234 (2011) 829-843.

797 [33] J.S. Chen, L.E. Mortenson, Purification and properties of hydrogenase from Clostridium

798 pasteurianum W5, Biochimica et Biophysica Acta-Protein Structure, 371 (1974) 283-298.

799 [34] P.M. Vignais, B. Billoud, J. Meyer, Classification and phylogeny of hydrogenases, Fems

800 Microbiology Reviews, 25 (2001) 455-501. 
801 [35] G.J. Schut, M.W.W. Adams, The iron-hydrogenase of Thermotoga maritima utilizes

802 ferredoxin and NADH synergistically: a new perspective on anaerobic hydrogen production,

803 Journal of Bacteriology, 191 (2009) 4451-4457.

804 [36] S. Wang, H. Huang, J. Kahnt, R.K. Thauer, A reversible electron-bifurcating ferredoxin and 805 NAD-dependent [FeFe]-hydrogenase (HydABC) in Moorella thermoacetica, Journal of 806 Bacteriology, 195 (2013) 1267-1275.

807 [37] Y. Zheng, J. Kahnt, I.H. Kwon, R.I. Mackie, R.K. Thauer, Hydrogen formation and its 808 regulation in Ruminococcus albus: involvement of an electron-bifurcating [FeFe]-hydrogenase, 809 of a non-electron-bifurcating [FeFe]-hydrogenase, and of a putative hydrogen-sensing [FeFe]810 hydrogenase, Journal of Bacteriology, 196 (2014) 3840-3852.

811 [38] G. Herrmann, E. Jayamani, G. Mai, W. Buckel, Energy conservation via electron812 transferring flavoprotein in anaerobic bacteria, Journal of Bacteriology, 190 (2008) 784-791.

813 [39] F. Li, J. Hinderberger, H. Seedorf, J. Zhang, W. Buckel, R.K. Thauer, Coupled ferredoxin 814 and crotonyl coenzyme A (CoA) reduction with NADH catalyzed by the butyryl-CoA 815 dehydrogenase/Etf complex from Clostridium kluyveri, Journal of Bacteriology, 190 (2008) 843816850.

817 [40] S. Wang, H. Huang, J. Kahnt, A.P. Mueller, M. Köpke, R.K. Thauer, NADP-specific 818 electron-bifurcating [FeFe]-hydrogenase in a functional complex with formate dehydrogenase in 819 Clostridium autoethanogenum grown on CO, Journal of Bacteriology, 195 (2013) 4373-4386.

820 [41] S. Wang, H. Huang, J. Kahnt, R.K. Thauer, Clostridium acidurici electron-bifurcating 821 formate dehydrogenase, Applied and Environmental Microbiology, 79 (2013) 6176-6179. 
822 [42] W. Buckel, R.K. Thauer, Energy conservation via electron bifurcating ferredoxin reduction 823 and proton/Na+ translocating ferredoxin oxidation, Biochimica Et Biophysica Acta-

824 Bioenergetics, 1827 (2013) 94-113.

825 [43] H.Y. Huang, S.N. Wang, J. Moll, R.K. Thauer, Electron bifurcation involved in the energy 826 metabolism of the acetogenic bacterium Moorella thermoacetica growing on glucose or $\mathrm{H}_{2}$ plus $827 \mathrm{CO}_{2}$, Journal of Bacteriology, 194 (2012) 3689-3699.

828 [44] S. Malki, I. Saimmaime, G. Deluca, M. Rousset, Z. Dermoun, J.P. Belaich, Characterization 829 of an operon encoding an NADP-reducing hydrogenase in Desulfovibrio fructosovorans, Journal 830 of Bacteriology, 177 (1995) 2628-2636.

831 [45] J.T. Henry, S. Crosson, Ligand-binding PAS domains in a genomic, cellular, and structural 832 context, Annual Review of Microbiology, 65 (2011) 261-286.

833 [46] F. Sievers, A. Wilm, D. Dineen, T.J. Gibson, K. Karplus, W. Li, R. Lopez, H. McWilliam, 834 M. Remmert, J. Söding, J.D. Thompson, D.G. Higgins, Fast, scalable generation of high-quality 835 protein multiple sequence alignments using Clustal Omega, Molecular Systems Biology, 7 836 (2011) 539-544.

837 [47] G.E. Crooks, G. Hon, J.M. Chandonia, S.E. Brenner, WebLogo: A Sequence Logo 838 Generator, Genome Research, 14 (2004) 1188-1190.

839 [48] I. Dondoshansky, Y. Wolf, Blastclust (ncbi software development toolkit), NCBI, Bethesda, 840 Md, (2002).

841 [49] A. Marchler-Bauer, M.K. Derbyshire, N.R. Gonzales, S. Lu, F. Chitsaz, L.Y. Geer, R.C. 842 Geer, J. He, M. Gwadz, D.I. Hurwitz, C.J. Lanczycki, F. Lu, G.H. Marchler, J.S. Song, N.

843 Thanki, Z. Wang, R.A. Yamashita, D. Zhang, C. Zheng, S.H. Bryant, CDD: NCBI's conserved 844 domain database, Nucleic Acids Research, 43 (2015) D222-D226. 
845 [50] The Universal Protein Resource (UniProt), Nucleic Acids Research, 36 (2008) D190-D195.

846 [51] M. Hahsler, S. Chelluboina, K. Hornik, C. Buchta, The arules R-package ecosystem:

847 Analyzing interesting patterns from large transaction data sets, Journal of Machine Learning

$848 \quad$ Research, 12 (2011) 2021-2025.

849 [52] R. Agrawal, R. Srikant, Fast algorithms for mining association rules, in: Proceedings of the 850 20th Very Large Databases (VLDB) Conference, vol. 1215, Santiago, Chile, 1994, pp. 487-499.

851 [53] O. Hammer, D.A.T. Harper, P.D. Ryan, PAST: Paleontological statistics software package

852 for education and data analysis, Palaeontologia Electronica, 4 (2001) 1-9.

853 [54] M.E. Smoot, K. Ono, J. Ruscheinski, P.L. Wang, T. Ideker, Cytoscape 2.8: New features for 854 data integration and network visualization, Bioinformatics, 27 (2011) 431-432.

855 [55] Y. Assenov, F. Ramirez, S.E. Schelhorn, T. Lengauer, M. Albrecht, Computing topological 856 parameters of biological networks, Bioinformatics, 24 (2008) 282-284.

857 [56] K. Ma, M.W.W. Adams, Sulfide dehydrogenase from the hyperthermophilic archaeon 858 Pyrococcus furiosus: a new multifunctional enzyme involved in the reduction of elemental 859 sulfur, Journal of Bacteriology, 176 (1994) 6509-6517.

860 [57] D.A. Ravcheev, X.Q. Li, H. Latif, K. Zengler, S.A. Leyn, Y.D. Korostelev, A.E. Kazakov, 861 P.S. Novichkov, A.L. Osterman, D.A. Rodionov, Transcriptional regulation of central carbon 862 and energy metabolism in bacteria by redox-responsive repressor Rex, Journal of Bacteriology, $863194(2012)$ 1145-1157.

864 [58] E. Hidalgo, B. Demple, An iron-sulfur center essential for transcriptional activation by the 865 redox-sensing SoxR protein, The EMBO Journal, 13 (1994) 138-146.

866 [59] M. Zheng, G. Storz, Redox sensing by prokaryotic transcription factors, Biochemical 867 Pharmacology, 59 (2000) 1-6. 
868 [60] S.N. Wang, H.Y. Huang, J. Moll, R.K. Thauer, NADP ${ }^{+}$reduction with reduced ferredoxin 869 and $\mathrm{NADP}^{+}$reduction with $\mathrm{NADH}$ are coupled via an electron-bifurcating enzyme complex in 870 Clostridium kluyveri, Journal of Bacteriology, 192 (2010) 5115-5123.

871 [61] P.M. Vignais, B. Billoud, Occurrence, classification, and biological function of 872 hydrogenases: An overview, Chemical Reviews, 107 (2007) 4206-4272.

873 [62] L. Feinberg, J. Foden, T. Barrett, K.W. Davenport, D. Bruce, C. Detter, R. Tapia, C. Han, 874 A. Lapidus, S. Lucas, J.F. Cheng, S. Pitluck, T. Woyke, N. Ivanova, N. Mikhailova, M. Land, L. 875 Hauser, D.A. Argyros, L. Goodwin, D. Hogsett, N. Caiazza, Complete genome sequence of the 876 cellulolytic thermophile Clostridium thermocellum DSM1313, Journal of Bacteriology, 193 877 (2011) 2906-2907.

878 [63] X. Brazzolotto, J.K. Rubach, J. Gaillard, S. Gambarelli, M. Atta, M. Fontecave, The [Fe879 Fe]-hydrogenase maturation protein HydF from Thermotoga maritima is a GTPase with an iron880 sulfur cluster, Journal of Biological Chemistry, 281 (2006) 769-774.

881 [64] E.M. Shepard, E.S. Boyd, J.B. Broderick, J.W. Peters, Biosynthesis of complex iron-sulfur 882 enzymes, Current Opinion in Chemical Biology, 15 (2011) 319-327.

883 [65] E.M. Shepard, B.R. Duffus, S.J. George, S.E. McGlynn, M.R. Challand, K.D. Swanson, 884 P.L. Roach, S.P. Cramer, J.W. Peters, J.B. Broderick, [FeFe]-hydrogenase maturation: HydG885 catalyzed synthesis of carbon monoxide, Journal of the American Chemical Society, 132 (2010) $8869247-9249$.

887 [66] E.M. Shepard, S.E. McGlynn, A.L. Bueling, C.S. Grady-Smith, S.J. George, M.A. Winslow, 888 S.P. Cramer, J.W. Peters, J.B. Broderick, Synthesis of the 2Fe subcluster of the [FeFe]889 hydrogenase H-cluster on the HydF scaffold, Proceedings of the National Academy of Sciences 890 of the United States of America, 107 (2010) 10448-10453. 
891 [67] J.K. Rubach, X. Brazzolotto, J. Gaillard, M. Fontecave, Biochemical characterization of the 892 HydE and HydG iron-only hydrogenase maturation enzymes from Thermatoga maritima, Febs 893 Letters, 579 (2005) 5055-5060.

894 [68] S. Bellon, J.D. Parsons, Y.Y. Wei, K. Hayakawa, L.L. Swenson, P.S. Charifson, J.A. 895 Lippke, R. Aldape, C.H. Gross, Crystal structures of Escherichia coli topoisomerase IV ParE 896 subunit (24 and 43 Kilodaltons): a single residue dictates differences in novobiocin potency 897 against topoisomerase IV and DNA gyrase, Antimicrobial Agents and Chemotherapy, 48 (2004) $898 \quad 1856-1864$.

899 [69] T. Tanaka, S.K. Saha, C. Tomomori, R. Ishima, D.J. Liu, K.I. Tong, H. Park, R. Dutta, L. 900 Qin, M.B. Swindells, T. Yamazaki, A.M. Ono, M. Kainosho, M. Inouye, M. Ikura, NMR 901 structure of the histidine kinase domain of the E. coli osmosensor EnvZ, Nature, 396 (1998) 8890292.

903 [70] L. Aravind, C.P. Ponting, The cytoplasmic helical linker domain of receptor histidine kinase 904 and methyl-accepting proteins is common to many prokaryotic signalling proteins, Fems 905 Microbiology Letters, 176 (1999) 111-116.

906 [71] A. Daddaoua, C. Molina-Santiago, J. de la Torre, T. Krell, J.L. Ramos, GtrS and GltR form 907 a two-component system: The central role of 2-ketogluconate in the expression of exotoxin A 908 and glucose catabolic enzymes in Pseudomonas aeruginosa, Nucleic Acids Research, 42 (2014) $909 \quad 7654-7665$.

910 [72] A. Marina, C.D. Waldburger, W.A. Hendrickson, Structure of the entire cytoplasmic portion 911 of a sensor histidine kinase protein, The EMBO Journal, 24 (2005) 4247-4259.

912 [73] P.M. Wolanin, P.A. Thomason, J.B. Stock, Histidine protein kinases: key signal transducers 913 outside the animal kingdom, Genome Biology, 3 (2002). 
914 [74] A.M. Stock, V.L. Robinson, P.N. Goudreau, Two-component signal transduction, Annual 915 Review of Biochemistry, 69 (2000) 183-215.

916 [75] K.E. Swain, J.J. Falke, Structure of the conserved HAMP domain in an intact, membrane917 bound chemoreceptor: a disulfide mapping study, Biochemistry, 46 (2007) 13684-13695.

918 [76] S.B. Williams, V. Stewart, Functional similarities among two-component sensors and 919 methyl-accepting chemotaxis proteins suggest a role for linker region amphipathic helices in 920 transmembrane signal transduction, Molecular Microbiology, 33 (1999) 1093-1102.

921 [77] B. Boxma, R.M. de Graaf, G.W.M. van der Staay, T.A. van Alen, G. Ricard, T. Gabaldon, 922 A.H.A.M. van Hoek, S.Y. Moon-van der Staay, W.J.H. Koopman, J.J. van Hellemond, A.G.M. 923 Tielens, T. Friedrich, M. Veenhuis, M.A. Huynen, J.H.P. Hackstein, An anaerobic 924 mitochondrion that produces hydrogen, Nature, 434 (2005) 74-79.

925 [78] B. Boxma, G. Ricard, A.H. van Hoek, E. Severing, S.Y. Moon-van der Staay, G.W.M. van 926 der Staay, T.A. van Alen, R.M. de Graaf, G. Cremers, M. Kwantes, N.R. McEwan, C.J. 927 Newbold, J.P. Jouany, T. Michalowski, P. Pristas, M.A. Huynen, J.H.P. Hackstein, The [FeFe] 928 hydrogenase of Nyctotherus ovalis has a chimeric origin, BMC Evolutionary Biology, 7 (2007) $929 \quad 230-242$.

930 [79] R.M. de Graaf, G. Ricard, T.A. van Alen, I. Duarte, B.E. Dutilh, C. Burgtorf, J.W.P. Kuiper, 931 G.W.M. van der Staay, A.G.M. Tielens, M.A. Huynen, J.H.P. Hackstein, The organellar genome 932 and metabolic potential of the hydrogen-producing mitochondrion of Nyctotherus ovalis, 933 Molecular Biology and Evolution, 28 (2011) 2379-2391.

934 [80] J. Cieśla, T. Frączyk, W. Rode, Phosphorylation of basic amino acid residues in proteins: 935 important but easily missed, ACTA Biochimica Polonica, 58 (2011) 137-147. 
936 [81] J. Deutscher, M.H. Saier, Ser/Thr/Tyr protein phosphorylation in bacteria - for long time 937 neglected, now well established, Journal of Molecular Microbiology and Biotechnology, 9 938 (2005) 125-131.

939 [82] S.F.F. Pereira, L. Goss, J. Dworkin, Eukaryote-like serine/threonine kinases and 940 phosphatases in Bacteria, Microbiology and Molecular Biology Reviews, 75 (2011) 192-212.

941 [83] J.B. Stock, A.J. Ninfa, A.M. Stock, Protein phosphorylation and regulation of adaptive 942 responses in Bacteria, Microbiological Reviews, 53 (1989) 450-490.

943 [84] M. Schnolzer, W.D. Lehmann, Identification of modified peptides by metastable 944 fragmentation in MALDI mass spectrometry, International Journal of Mass Spectrometry, 169 $945 \quad$ (1997) 263-271.

946 [85] A. Tholey, J. Reed, W.D. Lehmann, Electrospray tandem mass spectrometric studies of 947 phosphopeptides and phosphopeptide analogues, Journal of Mass Spectrometry, 34 (1999) 117 948123.

949 [86] D.T. McLachlin, B.T. Chait, Analysis of phosphorylated proteins and peptides by mass 950 spectrometry, Current Opinion in Chemical Biology, 5 (2001) 591-602.

951 [87] K.F. Medzihradszky, N.J. Phillipps, L. Senderowicz, P. Wang, C.W. Turck, Synthesis and 952 characterization of histidine-phosphorylated peptides, Protein Science, 6 (1997) 1405-1411.

953 [88] R.S. Annan, S.A. Carr, Phosphopeptide analysis by matrix-assisted laser desorption time-of954 flight mass spectrometry, Analytical Chemistry, 68 (1996) 3413-3421.

955 [89] S. Metzger, R. Hoffmann, Studies on the dephosphorylation of phosphotyrosine-containing 956 peptides during post-source decay in matrix-assisted laser desorption/ionization, Journal of Mass 957 Spectrometry, 35 (2000) 1165-1177. 
TABLE 1. Taxonomic distribution of HydA homologs in 2921 complete archaeal, bacterial, and eukaryal genomes. The total number of genomes for each phylum is indicated along with the number of genomes within that phylum that encode for HydA. The average number of HydA in genomes that encode HydA is indicated along with the number of HydA in each of the 3 defined Hyd groups (see results and discussion section for a description of the group designations). (Double column)

\begin{tabular}{|c|c|c|c|c|c|c|c|}
\hline \multirow[b]{2}{*}{ Domain } & \multirow[b]{2}{*}{ Phylum } & \multirow{2}{*}{$\begin{array}{l}\text { Total number } \\
\text { of genomes }\end{array}$} & \multirow{2}{*}{$\begin{array}{c}\text { Total number of } \\
\text { genomes with } \\
\text { HydA }\end{array}$} & \multirow{2}{*}{$\begin{array}{c}\text { Average \# } \\
\text { of HydA in } \\
\text { the } \\
\text { genomes }\end{array}$} & \multicolumn{3}{|c|}{ Total number of Hyd in each group } \\
\hline & & & & & Group 1 & Group 2 & Group 3 \\
\hline \multirow[t]{5}{*}{ Archaea } & Crenarchaeota & 50 & 0 & 0 & 0 & 0 & 0 \\
\hline & Euryarchaeota & 110 & 0 & 0 & 0 & 0 & 0 \\
\hline & Korarchaeota & 1 & 0 & 0 & 0 & 0 & 0 \\
\hline & Nanoarchaeota & 1 & 0 & 0 & 0 & 0 & 0 \\
\hline & Thaumarchaeota & 5 & 0 & 0 & 0 & 0 & 0 \\
\hline \multirow[t]{30}{*}{ Bacteria } & Acidobacteria & 7 & 0 & 0 & 0 & 0 & 0 \\
\hline & Actinobacteria & 301 & 6 & 1.5 & 9 & 0 & 0 \\
\hline & Aquificae & 14 & 1 & 1 & 1 & 0 & 0 \\
\hline & Armatimonadetes & 2 & 0 & 0 & 0 & 0 & 0 \\
\hline & Bacteroidetes & 94 & 10 & 1.5 & 11 & 2 & 2 \\
\hline & Caldiserica & 1 & 0 & 0 & 0 & 0 & 0 \\
\hline & Chlamydia & 109 & 0 & 0 & 0 & 0 & 0 \\
\hline & Chlorobi & 11 & 0 & 0 & 0 & 0 & 0 \\
\hline & Chloroflexi & 24 & 8 & 1 & 0 & 8 & 0 \\
\hline & Chrysiogenetes & 1 & 0 & 0 & 0 & 0 & 0 \\
\hline & Cloacimonetes & 1 & 1 & 1 & 0 & 0 & 1 \\
\hline & Cyanobacteria & 79 & 0 & 0 & 0 & 0 & 0 \\
\hline & Deferribacteres & 4 & 0 & 0 & 0 & 0 & 0 \\
\hline & Deinococcus-Thermus & 21 & 0 & 0 & 0 & 0 & 0 \\
\hline & Dictyoglomi & 2 & 2 & 2 & 2 & 2 & 0 \\
\hline & Elusimicrobia & 1 & 1 & 1 & 0 & 1 & 0 \\
\hline & Fibrobacteres & 2 & 0 & 0 & 0 & 0 & 0 \\
\hline & Firmicutes & 622 & 165 & 2.9 & 347 & 92 & 52 \\
\hline & Fusobacteria & 11 & 2 & 4.5 & 4 & 5 & 0 \\
\hline & Gemmatimonadetes & 1 & 0 & 0 & 0 & 0 & 0 \\
\hline & Ignavibacteriae & 2 & 2 & 3.5 & 2 & 5 & 0 \\
\hline & Nitrospirae & 4 & 1 & 2 & 2 & 0 & 0 \\
\hline & Planctomycetes & 8 & 0 & 0 & 0 & 0 & 0 \\
\hline & Proteobacteria & 1240 & 39 & 1.5 & 44 & 7 & 6 \\
\hline & Spirochaetes & 65 & 22 & 2.5 & 31 & 14 & 11 \\
\hline & Synergistetes & 5 & 2 & 1 & 0 & 1 & 1 \\
\hline & Tenericutes & 89 & 0 & 0 & 0 & 0 & 0 \\
\hline & Thermodesulfobacteria & 2 & 1 & 4 & 4 & 0 & 0 \\
\hline & Thermotogae & 18 & 17 & 2.3 & 20 & 16 & 3 \\
\hline & Verrucomicrobia & 5 & 1 & 1 & 0 & 1 & 0 \\
\hline \multirow[t]{9}{*}{ Eukaryota } & Heterokonts & 1 & 1 & 1 & 1 & 0 & 0 \\
\hline & Chlorophyta & 3 & 3 & 1.7 & 5 & 0 & 0 \\
\hline & Ciliophora & 1 & 1 & 1 & 0 & 1 & 0 \\
\hline & Ascomycota & 14 & 0 & 0 & 0 & 0 & 0 \\
\hline & Microsporidia & 1 & 0 & 0 & 0 & 0 & 0 \\
\hline & Euglenozoa & 1 & 0 & 0 & 0 & 0 & 0 \\
\hline & Mognoliophyta & 1 & 0 & 0 & 0 & 0 & 0 \\
\hline & Chordata & 2 & 0 & 0 & 0 & 0 & 0 \\
\hline & Nematoda & 1 & 0 & 0 & 0 & 0 & 0 \\
\hline
\end{tabular}




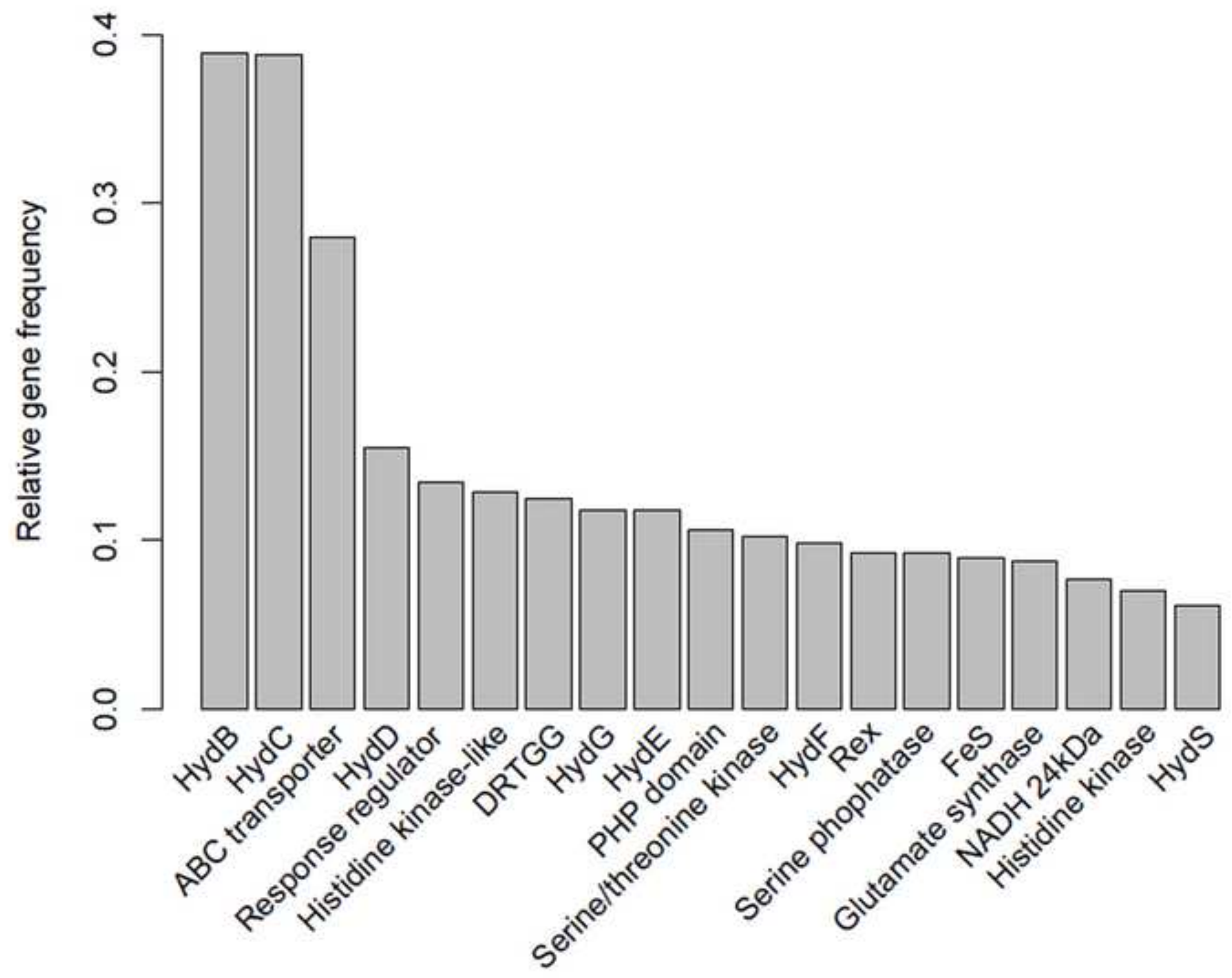




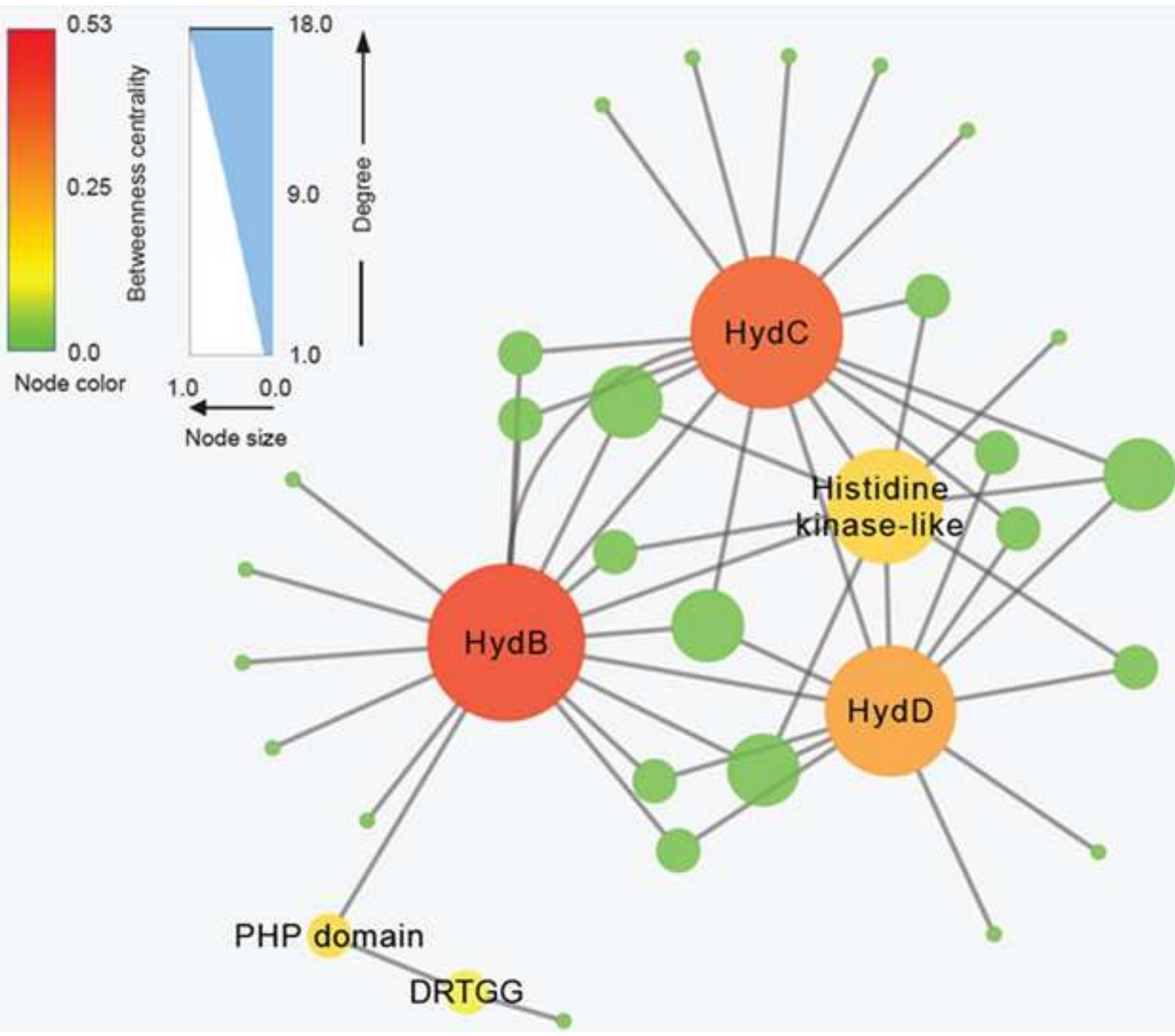



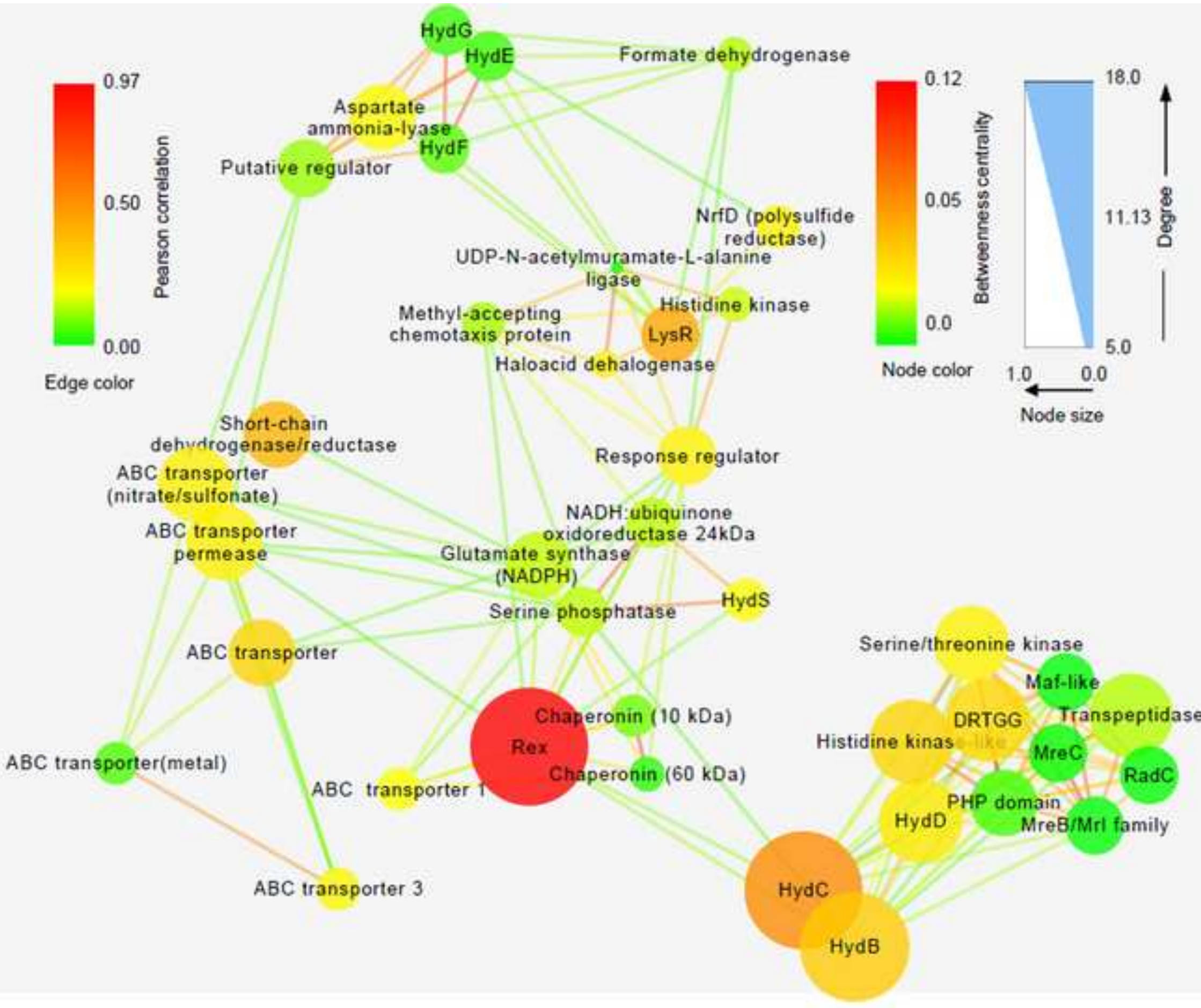


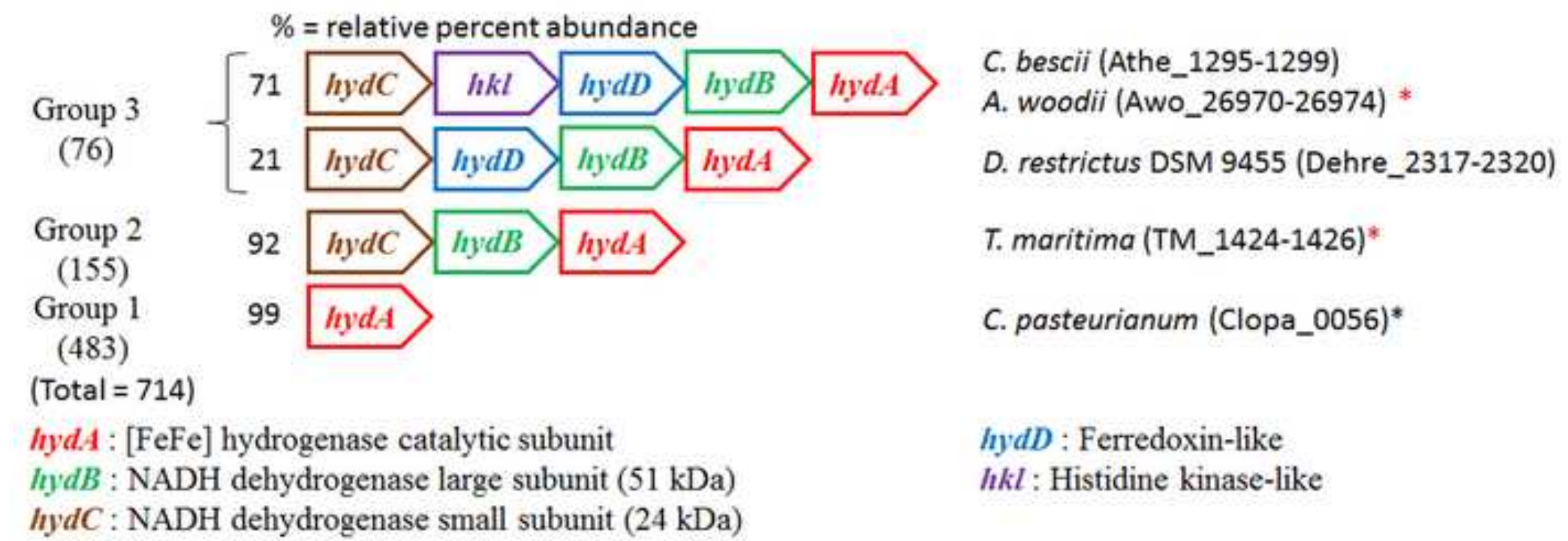




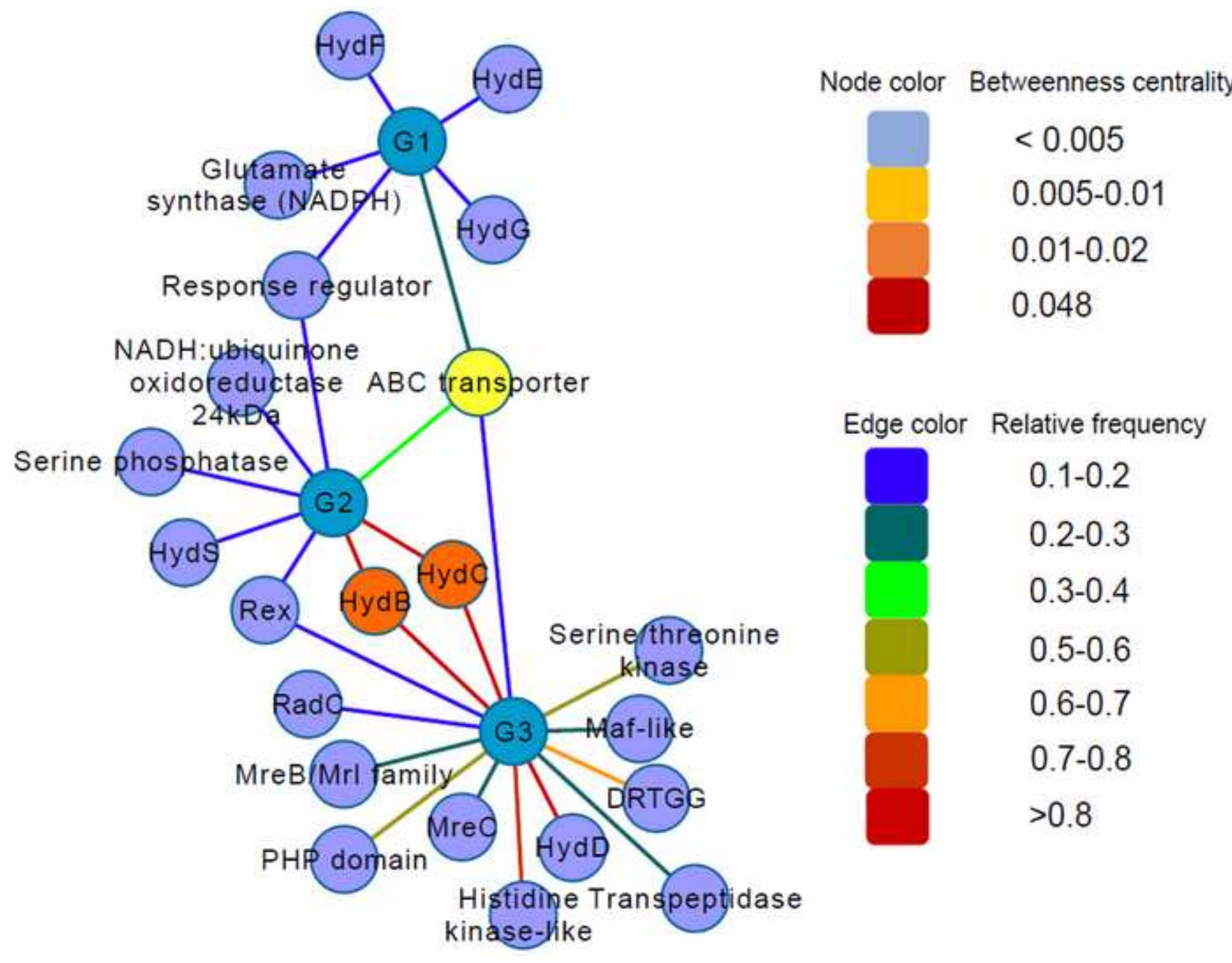




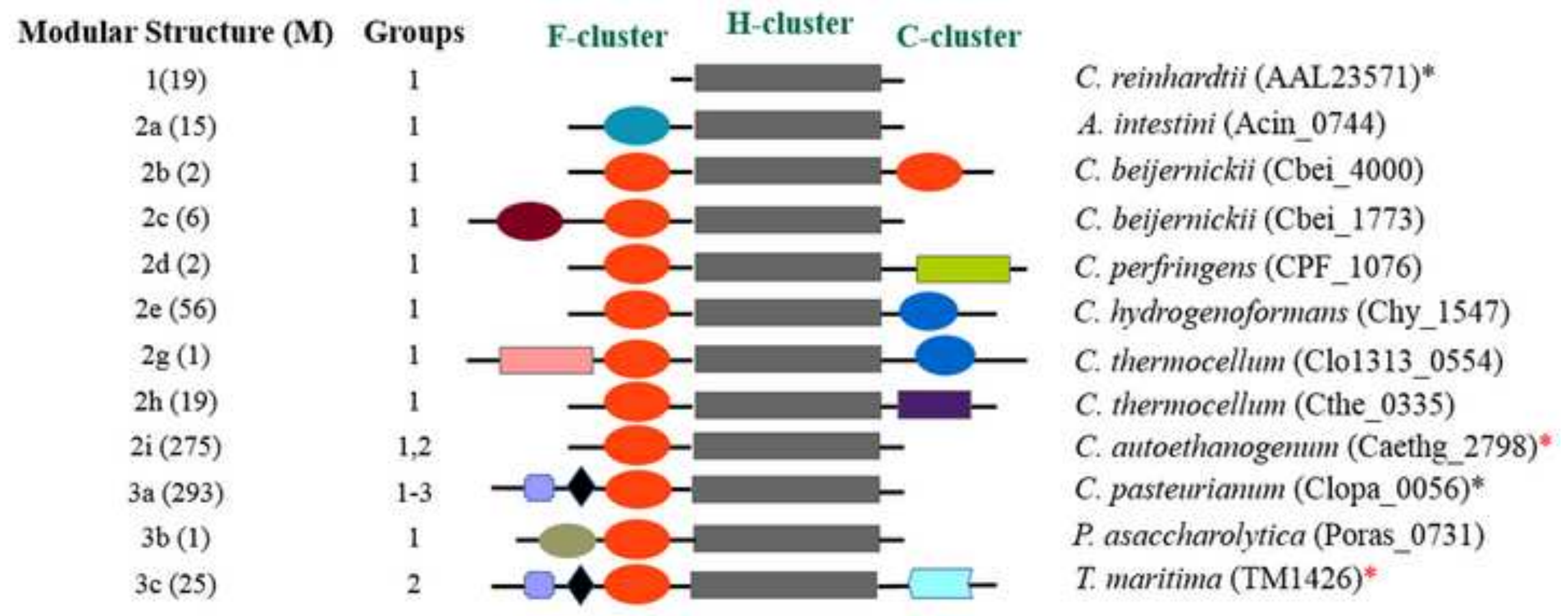

D NADH quinone oxidoreductase subunit $\mathrm{F}$

$\mathrm{CO}$ dehydrogenase/acetyl-CoA synthase gamma subunit (CdhE)

$\square$ Rubredoxin-rubrerythrin
FeS cluster $\mathrm{FeS}\left(\mathrm{Cx}_{1-4} \mathrm{Cx}_{5-9} \mathrm{Cx}_{3} \mathrm{C}\right)$ Glutamate synthase Thioredoxin like
> $[4 \mathrm{Fe}-4 \mathrm{~S}](\mathrm{HCCC})$

ㄴ. $[2 \mathrm{Fe}-2 \mathrm{~S}]$ $2[4 \mathrm{Fe}-4 \mathrm{~S}]$ 8 Cys run 


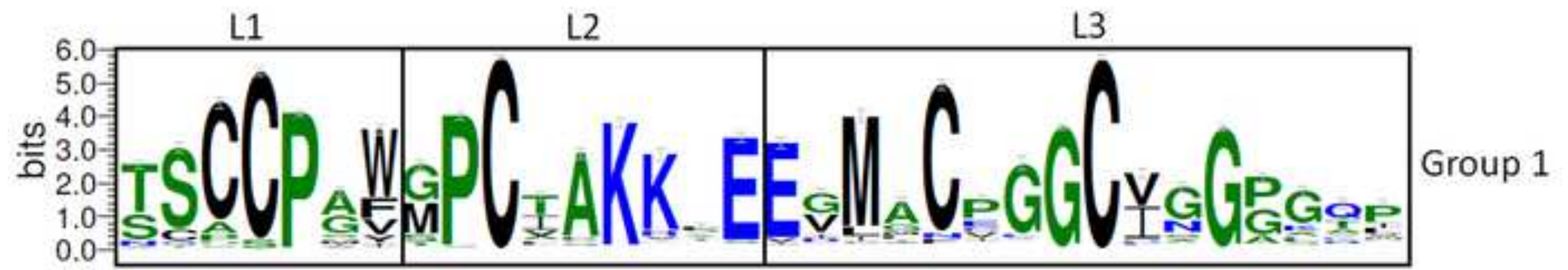

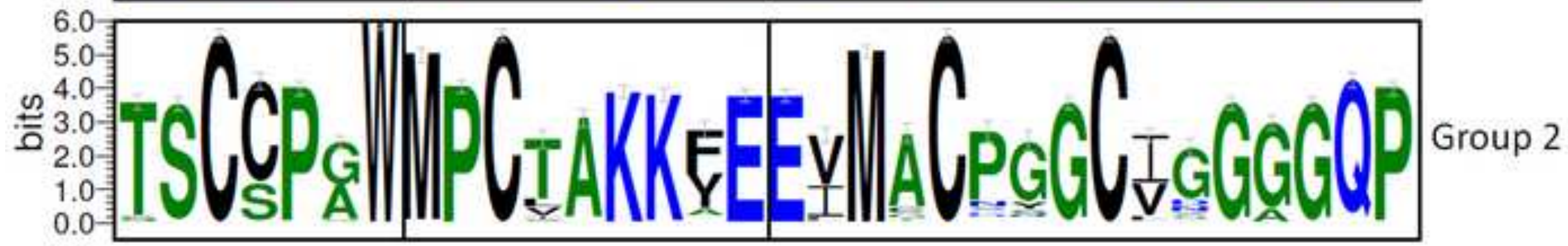

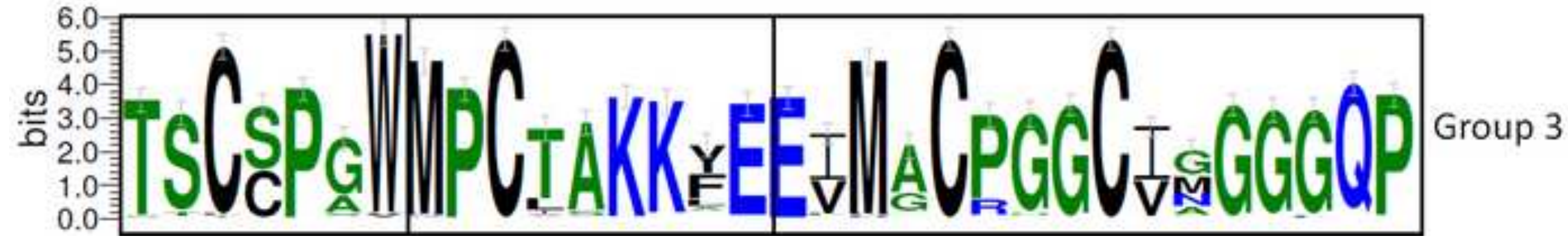



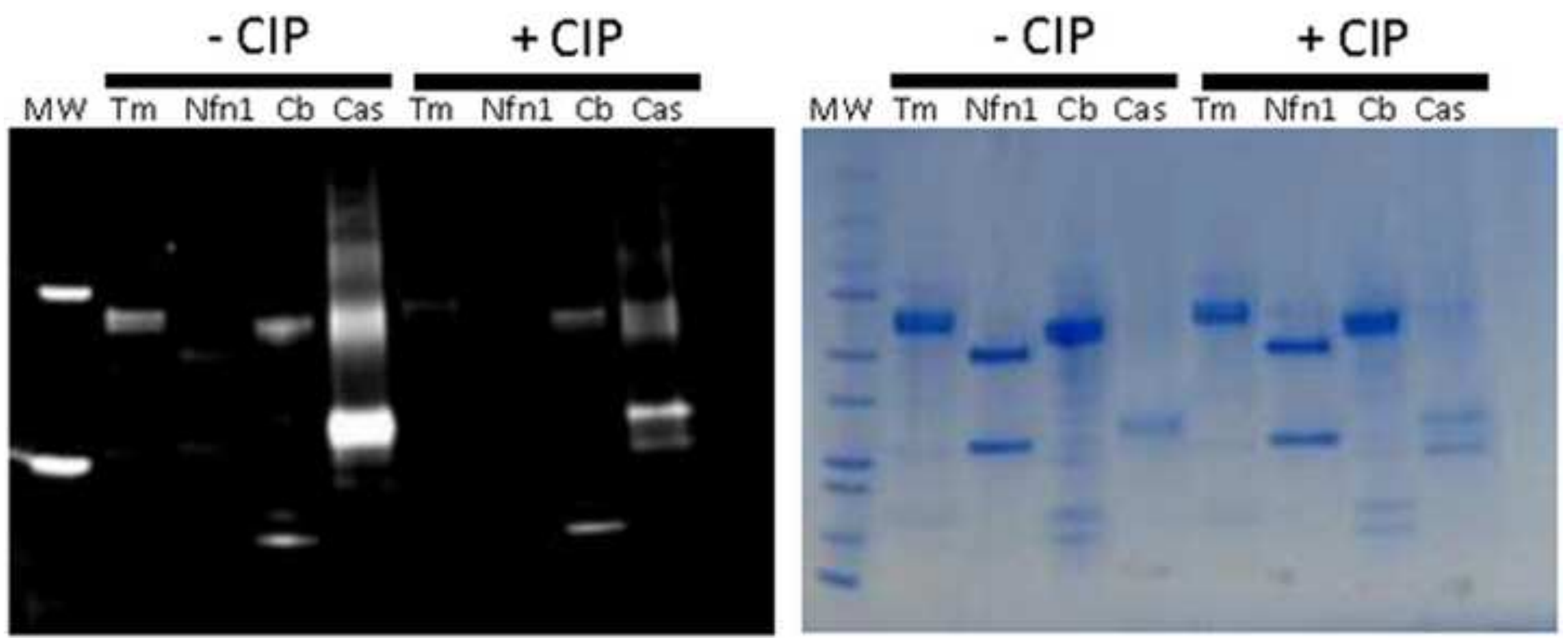
Figure 9
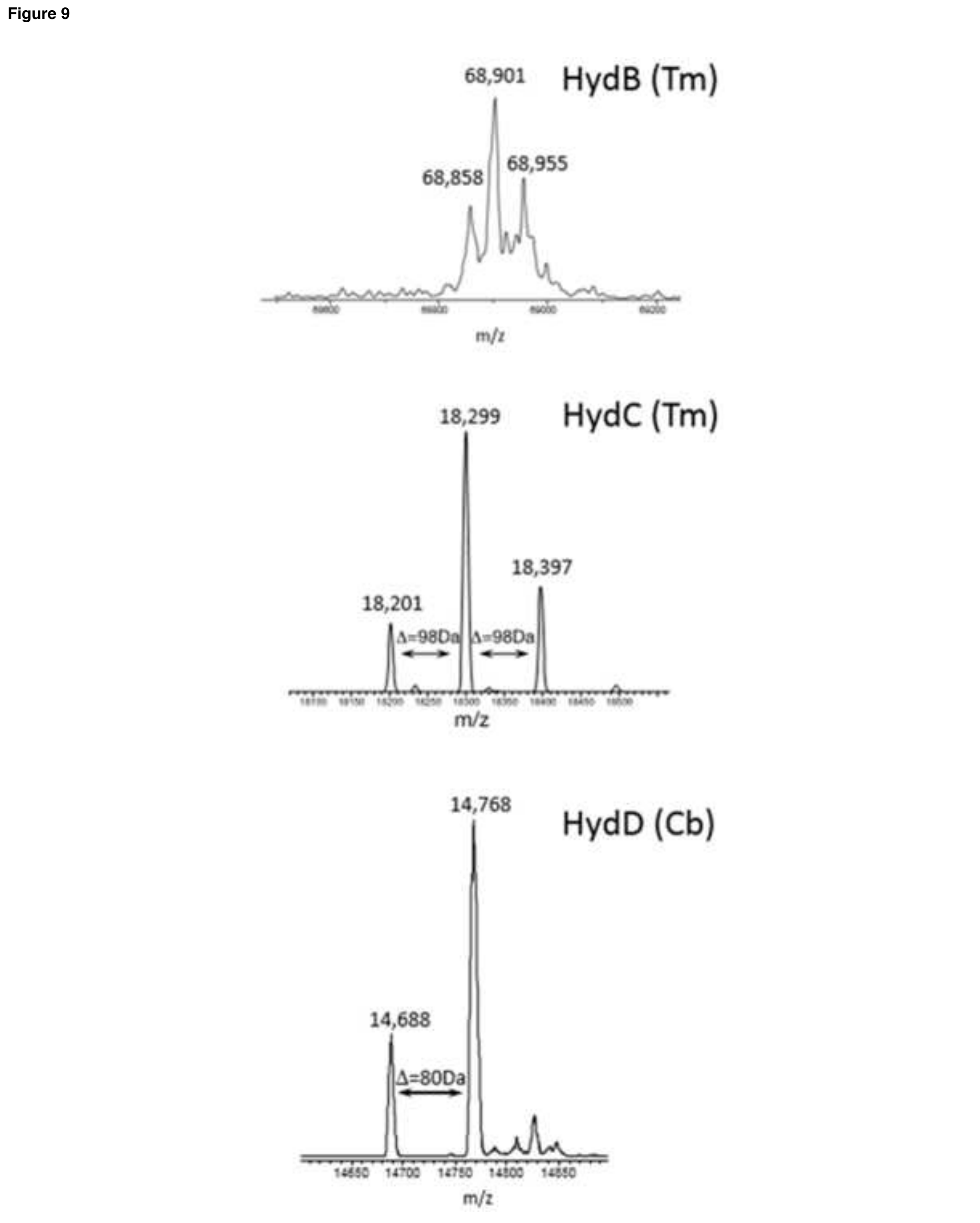

- 